\title{
DIRECT Distances to Nearby Galaxies Using Detached Eclipsing Binaries and Cepheids. III. Variables in the Field M31C
}

\author{
K. Z. Stanek ${ }^{2}$ \\ Harvard-Smithsonian Center for Astrophysics, 60 Garden St., MS20, Cambridge, MA 02138 \\ e-mail: kstanek@cfa.harvard.edu \\ J. Kaluzny \\ Warsaw University Observatory, Al. Ujazdowskie 4, PL-00-478 Warszawa, Poland \\ e-mail: jka@sirius.astrouw.edu.pl \\ M. Krockenberger, D. D. Sasselov \\ Harvard-Smithsonian Center for Astrophysics, 60 Garden St., MS16, Cambridge, MA 02138 \\ e-mail: krocken@cfa.harvard.edu, sasselov@cfa.harvard.edu \\ J. L. Tonry \\ University of Hawaii, Institute for Astronomy, 2680 Woodlawn Dr., Honolulu, HI 96822 \\ e-mail: jt@avidya.ifa.hawaii.edu \\ M. Mateo \\ Department of Astronomy, University of Michigan, 821 Dennison Bldg., Ann Arbor, \\ MI 48109-1090 \\ e-mail: mateo@astro.lsa.umich.edu
}

\begin{abstract}
We undertook a long term project, DIRECT, to obtain the direct distances to two important galaxies in the cosmological distance ladder - M31 and M33 - using detached eclipsing binaries (DEBs) and Cepheids. While rare and difficult to detect, DEBs provide us with the potential to determine these distances with an accuracy better than $5 \%$. The extensive photometry obtained in order to detect DEBs provides us with good light curves for the Cepheid variables. These are essential to the parallel project to derive direct Baade-Wesselink distances to Cepheids in M31 and M33. For both Cepheids and eclipsing binaries, the distance estimates will be free of any intermediate steps.

As a first step in the DIRECT project, between September 1996 and October 1997 we obtained 95 full/partial nights on the F. L. Whipple Observatory $1.2 \mathrm{~m}$ telescope
\end{abstract}

\footnotetext{
${ }^{1}$ Based on the observations collected at the Michigan-Dartmouth-MIT (MDM) $1.3 \mathrm{~m}$ telescope and at the F. L. Whipple Observatory (FLWO) $1.2 \mathrm{~m}$ telescope

${ }^{2}$ On leave from N. Copernicus Astronomical Center, Bartycka 18, Warszawa PL-00-716, Poland
} 
and 36 full nights on the Michigan-Dartmouth-MIT $1.3 \mathrm{~m}$ telescope to search for DEBs and new Cepheids in the M31 and M33 galaxies. In this paper, third in the series, we present the catalog of variable stars, most of them newly detected, found in the field M31C $[(\alpha, \delta)=(11.10,41.42), \mathrm{J} 2000.0]$. We have found 115 variable stars: 12 eclipsing binaries, 35 Cepheids and 68 other periodic, possible long period or non-periodic variables. The catalog of variables, as well as their photometry and finding charts, is available via anonymous ftp and the World Wide Web. The complete set of the CCD frames is available upon request.

Subject headings: binaries: eclipsing — Cepheids — distance scale — galaxies: individual (M31) — stars: variables: other

\section{Introduction}

The two nearby galaxies M31 and M33 are stepping stones to most of our current effort to understand the evolving universe at large scales. First, they are essential to the calibration of the extragalactic distance scale (Jacoby et al. 1992; Tonry et al. 1997). Second, they constrain population synthesis models for early galaxy formation and evolution and provide the stellar luminosity calibration. There is one simple requirement for all this - accurate distances.

Detached eclipsing binaries (DEBs) have the potential to establish distances to M31 and M33 with an unprecedented accuracy of better than $5 \%$ and possibly to better than $1 \%$. These distances are now known to no better than 10-15\%, as there are discrepancies of $0.2-0.3$ mag between RR Lyrae and Cepheids distance indicators (e.g. Huterer, Sasselov \& Schechter 1995; Holland 1998; Stanek \& Garnavich 1998). Detached eclipsing binaries (for reviews see Andersen 1991; Paczyński 1997) offer a single step distance determination to nearby galaxies and may therefore provide an accurate zero point calibration - a major step towards very accurate determination of the Hubble constant, presently an important but daunting problem for astrophysicists.

The detached eclipsing binaries have yet to be used (Huterer et al. 1995; Hilditch 1996) as distance indicators to M31 and M33. According to Hilditch (1996), there were about 60 eclipsing binaries of all kinds known in M31 (Gaposchkin 1962; Baade \& Swope 1963, 1965) and only one in M33 (Hubble 1929). Only now does the availability of large-format CCD detectors and inexpensive CPUs make it possible to organize a massive search for periodic variables, which will produce a handful of good DEB candidates. These can then be spectroscopically followed-up with the powerful new 6.5-10 meter telescopes.

The study of Cepheids in M31 and M33 has a venerable history (Hubble 1926, 1929; Gaposchkin 1962; Baade \& Swope 1963, 1965). In the 1980s, Freedman \& Madore (1990) and Freedman, Wilson, \& Madore (1991) studied small samples of the earlier discovered Cepheids, to build period-luminosity (P-L) relations in M31 and M33, respectively. However, both the 
sparse photometry and the small samples do not provide a good basis for obtaining direct Baade-Wesselink distances (see, e.g., Krockenberger, Sasselov \& Noyes 1997) to Cepheids-the need for new digital photometry has been long overdue. Recently, Magnier et al. (1997) surveyed large portions of M31, which have previously been ignored, and found some 130 new Cepheid variable candidates. Their light curves are, however, rather sparsely sampled and in the $V$-band only.

In Kaluzny et al. (1998, hereafter: Paper I) and Stanek et al. (1998, hereafter: Paper II), the first two papers of the series, we presented the catalogs of variable stars found in two fields in M31, called M31B and M31A. Here we present the catalog of variables from the next field M31C. In Sec. 2 we discuss the selection of the fields in M31 and the observations. In Sec.3 we describe the data reduction and calibration. In Sec.4 we discuss briefly the automatic selection we used for finding the variable stars. In Sec.5 we discuss the classification of the variables. In Sec. 6 we present the catalog of variable stars, followed by brief discussion of the results in Sec.7.

\section{Fields selection and observations}

M31 was primarily observed with the $1.3 \mathrm{~m}$ McGraw-Hill Telescope at the MichiganDartmouth-MIT (MDM) Observatory. We used the front-illuminated, Loral $2048^{2}$ CCD "Wilbur" (Metzger, Tonry \& Luppino 1993), which at the $f / 7.5$ station of the $1.3 \mathrm{~m}$ telescope has a pixel scale of 0.32 arcsec pixel $^{-1}$ and field of view of roughly 11 arcmin. We used Kitt Peak Johnson-Cousins BVI filters. Data for M31 were also obtained, mostly in 1997, with the $1.2 \mathrm{~m}$ telescope at the F. L. Whipple Observatory (FLWO), where we used "AndyCam" (Szentgyorgyi et al. 1998), with a thinned, back-side illuminated, AR coated Loral $2048^{2}$ pixel CCD. The pixel scale happens to be essentially the same as at the MDM $1.3 \mathrm{~m}$ telescope. We used standard Johnson-Cousins BVI filters.

Fields in M31 were selected using the MIT photometric survey of M31 by Magnier et al. (1992) and Haiman et al. (1994) (see Paper I, Fig.1). We selected six $11^{\prime} \times 11^{\prime}$ fields, M31A-F, four of them (A-D) concentrated on the rich spiral arm in the northeast part of M31, one (E) coinciding with the region of M31 searched for microlensing by Crotts \& Tomaney (1996), and one (F) containing the giant star formation region known as NGC206 (observed by Baade \& Swope 1963). Fields A-C were observed during September and October 1996 five to eight times per night in the $V$ band, resulting in total of 110-160 $V$ exposures per field. Fields D-F were observed once a night in the $V$-band. Some exposures in $B$ and $I$ were also taken. M31 was also observed, in 1996 and 1997, at the FLWO $1.2 \mathrm{~m}$ telescope, whose main target was M33.

In this paper we present the results for the M31C field. We obtained for this field useful data during 29 nights at the MDM, collecting a total of $141 \times 900 \mathrm{sec}$ exposures in $V$ and $30 \times 600 \mathrm{sec}$ exposures in $I$. We also obtained for this field useful data during 24 nights at the FLWO, in 1996 and 1997, collecting a total of $20 \times 900 \mathrm{sec}$ exposures in $V, 25 \times 600 \mathrm{sec}$ exposures in $I$ and 
$10 \times 1200 \mathrm{sec}$ exposures of $B$.

\section{Data reduction, calibration and astrometry}

The details of the reduction procedure were given in Paper I. Preliminary processing of the CCD frames was done with the standard routines in the IRAF-CCDPROC package.f Stellar profile photometry was extracted using the Daophot/Allstar package (Stetson 1987, 1992). We selected a "template" frame for each filter using a single frame of particularly good quality. These template images were reduced in a standard way (Paper I). Other images were reduced using Allstar in the fixed-position-mode using as an input the transformed object list from the template frames. For each frame the list of instrumental photometry derived for a given frame was transformed to the common instrumental system of the appropriate "template" image. Photometry obtained for the $B, V$ and $I$ filters was combined into separate data bases. M31C images obtained at the FLWO were reduced using MDM "templates". In case of $B$-band images obtained at FLWO we used the $V$-band MDM template to fix the positions of the stars.

The photometric VI calibration of the MDM data was discussed in Paper I. In addition, for the field M31C on the night of 1997 October 9/10 we have obtained independent $B V I$ calibration with the FLWO $1.2 \mathrm{~m}$ telescope. There was an offset of $0.012 \mathrm{mag}$ in $V$ and $0.024 \mathrm{mag}$ in $V-I$ between the FLWO and the MDM calibration, i.e. well within our estimate of the total $0.05 \mathrm{mag}$ systematic error discussed in Paper I. We also derived equatorial coordinates for all objects included in the data bases for the $V$ filter. The transformation from rectangular coordinates to equatorial coordinates was derived using $\sim 200$ stars identified in the list published by Magnier et al. (1992).

\section{Selection of variables}

The procedure for selecting the variables was described in detail in Paper I, so here we only give a short description, noting changes when necessary. The reduction procedure described in previous section produces databases of calibrated $B V I$ magnitudes and their standard errors. The $B V$ databases for M31C field contain 15120 stars, with up to 161 measurements in $V$ and up to 10 measurements in $B$, and the $I$ database contains 28441 stars with up to 55 measurements.

\footnotetext{
${ }^{3}$ The complete list of exposures for this field and related data files are available through anonymous ftp on cfa-ftp.harvard.edu, in pub/kstanek/DIRECT directory. Please retrieve the README file for instructions. Additional information on the DIRECT project is available through the wW at http://cfa-www.harvard.edu/ ${ }^{\sim k s t a n e k / D I R E C T / \text {. }}$

${ }^{4}$ IRAF is distributed by the National Optical Astronomy Observatories, which are operated by the Associations of Universities for Research in Astronomy, Inc., under cooperative agreement with the NSF
} 


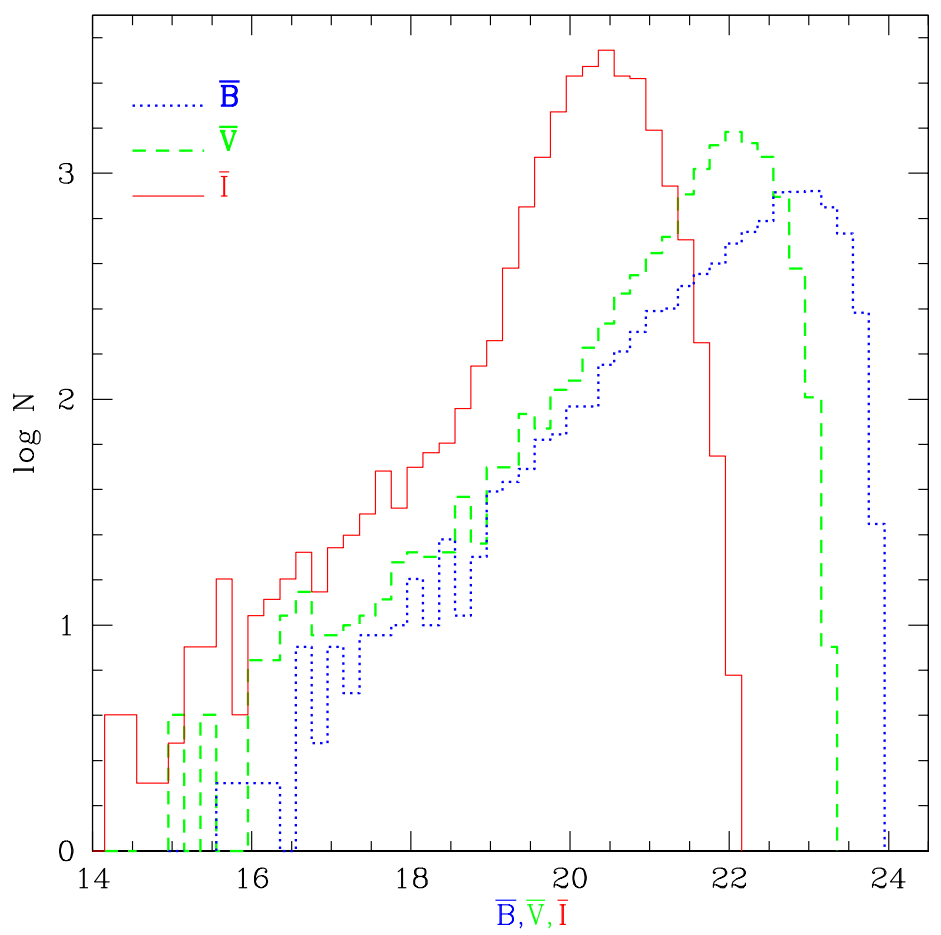

Fig. 1.- Distributions in $B$ (dotted line), $V$ (dashed line) and $I$ (continuous line) of stars in the field M31C.

Figure 1 shows the distributions of stars as a function of mean $\bar{B}, \bar{V}$ or $\bar{I}$ magnitude. As can be seen from the shape of the histograms, our completeness starts to drop rapidly at about $\bar{B} \sim 23$, $\bar{V} \sim 22$ and $\bar{I} \sim 20.5$. The primary reason for this difference in the depth of the photometry between $B V$ and $I$ is the level of the combined sky and background light, which is about three times higher in the $I$ filter than in the $B V$ filters.

The measurements flagged as "bad" and measurements with errors exceeding the average error by more than $4 \sigma$ are removed (Paper I). Usually zero to 10 points are removed, leaving the majority of stars with roughly $N_{\text {good }} \sim 150-160 \mathrm{~V}$ measurements. For further analysis we use only those stars that have at least $N_{\text {good }}>N_{\max } / 2(=80)$ measurements. There are 11263 such stars in the $V$ database of the M31C field.

Our next goal is to select objectively a sample of variable stars from the total sample defined above. There are many ways to proceed, and we largely follow the approach of Stetson (1996). The procedure is described in more detail in Paper I. In short, for each star we compute the Stetson's variability index $J_{S}$ (Paper I, Eq.7), and stars with values exceeding some minimum value $J_{S, \min }$ are considered candidate variables. The definition of Stetson's variability index includes the standard errors of individual observations. If, for some reason, these errors were overor underestimated, we would either miss real variables, or select spurious variables as real ones. Using the procedure described in Paper I, we scale the Daophot errors to better represent the "true" photometric errors. We then select the candidate variable stars by computing the value of 
$-6-$

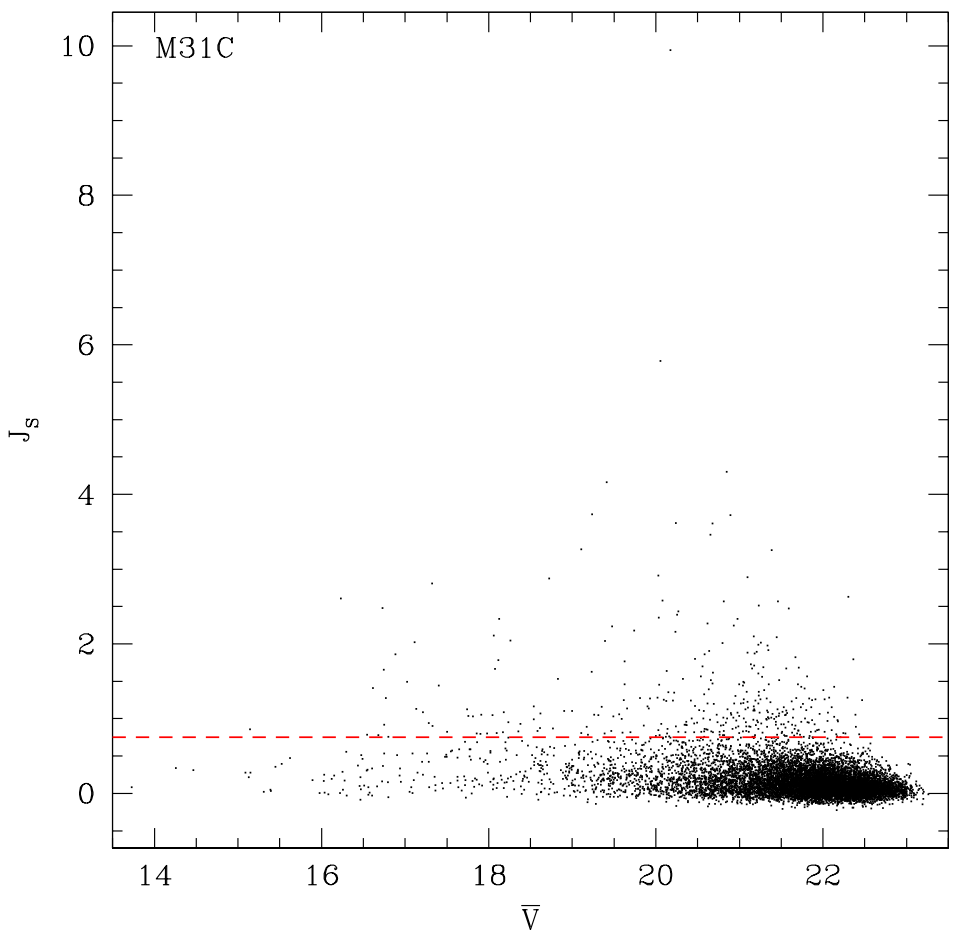

Fig. 2.- Variability index $J_{S}$ vs. mean $\bar{V}$ magnitude for 11262 stars in the field M31C with $N_{\text {good }}>80$. Dashed line at $J_{S}=0.75$ defines the cutoff applied for variability.

$J_{S}$ for the stars in our $V$ database. We used a cutoff of $J_{S, \min }=0.75$ and additional cuts described in Paper I to select 313 candidate variable stars (about $3 \%$ of the total number of 11263). In Figure 2 we plot the variability index $J_{S}$ vs. apparent visual magnitude $\bar{V}$ for 11262 stars with $N_{\text {good }}>80$.

\section{Period determination, classification of variables}

We based our candidate variables selection on the $V$ band data collected at the MDM and the FLWO telescopes. We also have the $B I$-bands data for the field, up to $55 I$-band epochs and up to $10 B$-band epochs, although for a variety of reasons some of the candidate variable stars do not have an $B$ or $I$-band counterpart. We will therefore not use the $B I$ data for the period determination and broad classification of the variables. We will however use the $B I$ data for the "final" classification of some variables.

Next we searched for the periodicities for all 313 candidate variables, using a variant of the Lafler-Kinman (1965) technique proposed by Stetson (1996). Starting with the minimum period of 0.25 days, successive trial periods are chosen so

$$
P_{j+1}^{-1}=P_{j}^{-1}-\frac{0.02}{\Delta t}
$$

where $\Delta t=t_{N}-t_{1}=398$ days is the time span of the series. The maximum period considered is 
150 days. For each candidate variable 10 best trial periods are selected (Paper I) and then used in our classification scheme.

The variables we are most interested in are Cepheids and eclipsing binaries (EBs). We therefore searched our sample of variable stars for these two classes of variables. As mentioned before, for the broad classification of variables we restricted ourselves to the $V$ band data. We will, however, present and use the $B I$-bands data, when available, when discussing some of the individual variable stars.

For EBs we used search strategy described in Paper II. Within our assumption the light curve of an EB is determined by nine parameters: the period, the zero point of the phase, the eccentricity, the longitude of periastron, the radii of the two stars relative to the binary separation, the inclination angle, the fraction of light coming from the bigger star and the uneclipsed magnitude. A total of 17 variables passed all of the criteria. We then went back to the CCD frames and tried to see by eye if the inferred variability is indeed there, especially in cases when the light curve is very noisy/chaotic. We decided to remove five dubious eclipsing binaries. The remaining 12 EBs with their parameters and light curves are presented in the Section 6.1.

In the search for Cepheids we followed the approach by Stetson (1996) of fitting template light curves to the data. We used the parameterization of Cepheid light curves in the $V$-band as given by Stetson (1996). There was a total of 100 variables passing all of the criteria (Paper I and II), but after investigating the CCD frames we removed 28 dubious "Cepheids", which leaves us with 62 probable Cepheids. Their parameters and light curves are presented in the Sections 6.2, 6.3.

After the preliminary selection of 17 eclipsing binaries and 100 possible Cepheids, we were left with 197 "other" variable stars. After raising the threshold of the variability index to $J_{S, \min }=1.2$ (Paper I) we are left with 61 variables. After investigating the CCD frames we removed 30 dubious variables from the sample, which leaves 31 variables which we classify as miscellaneous. Their parameters and light curves are presented in the Section 6.4.

\section{Catalog of variables}

In this section we present light curves and some discussion of the 115 variable stars discovered by our survey in the field M31C. P The variable stars are named according to the following convention: letter $\mathrm{V}$ for "variable", the number of the star in the $V$ database, then the letter "D" for our project, DIRECT, followed by the name of the field, in this case (M)31C, e.g. V9037 D31C. Tables 1, 2, 3 and 1 list the variable stars sorted broadly by four categories: eclipsing binaries, Cepheids, other periodic variables and "miscellaneous" variables, in our case meaning

\footnotetext{
${ }^{5}$ Complete $V$ and (when available) $B I$ photometry and $128 \times 128$ pixel $\left(\sim 40^{\prime \prime} \times 40^{\prime \prime}\right) V$ finding charts for all variables are available from the authors via the anonymous ftp from the Harvard-Smithsonian Center for Astrophysics and can be also accessed through the World Wide Web.
} 
"variables with no clear periodicity". Some of the variables which were found independently by survey of Magnier et al. (1997) are denoted in the "Comments" by "Ma97 ID", where the "ID" is the identification number assigned by Magnier at al. (1997). We also cross-identify several variables found by us in Paper I.

\subsection{Eclipsing binaries}

In Table 1 we present the parameters of the 12 eclipsing binaries in the M31C field. The lightcurves of these variables are shown in Figure 3, along with the simple eclipsing binary models discussed in the Paper I. The variables are sorted in the Table 1 by the increasing value of the period $P$. For each eclipsing binary we present its name, J2000.0 coordinates (in degrees), period $P$, magnitudes $V_{\max }, I_{\max }$ and $B_{\max }$ of the system outside of the eclipse, and the radii of the binary components $R_{1}, R_{2}$ in the units of the orbital separation. We also give the inclination angle of the binary orbit to the line of sight $i$ and the eccentricity of the orbit $e$. The reader should bear in mind that the values of $V_{\max }, I_{\max }, B_{\max }, R_{1}, R_{2}, i$ and $e$ are derived with a straightforward model of the eclipsing system, so they should be treated only as reasonable estimates of the "true" value.

One of the eclipsing binaries found, V9037 D31C, is a very good DEB candidate, with deep eclipses and the ellipticity indicating that the system is young and unevolved. However, much better light curve is necessary to accurately establish the properties of the system. Two other systems, V10732 and V14662 D31C, also seem to be detached, but they are significantly fainter than V9037 D31C and therefore less suitable for follow-up.

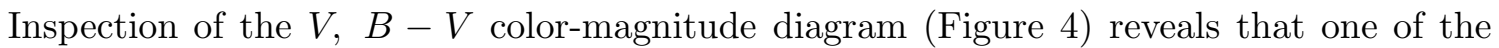
candidate eclipsing binaries lands close to the Cepheid portion of the CMD. It turns out that this variable, V14396 D31C, is only marginally better fit by a eclipsing binary light curve than by a Cepheid light curve with roughly half of the period, but we decided to keep it classified as an eclipsing binary.

\subsection{Cepheids}

In Table 2 we present the parameters of 35 Cepheids in the M31C field, sorted by the period $P$. For each Cepheid we present its name, J2000.0 coordinates, period $P$, flux-weighted mean magnitudes $\langle V\rangle$ and (when available) $\langle I\rangle$ and $\langle B\rangle$, and the $V$-band amplitude of the variation $A$. In Figure 5 we show the phased $B, V, I$ lightcurves of our Cepheids. Also shown is the best fit template lightcurve (Stetson 1996), which was fitted to the $V$ data and then for the $I$ data only the zero-point offset was allowed. For the $B$-band data, lacking the template lightcurve parameterization (Stetson 1996), we used the $V$-band template, allowing for different zero-points and amplitudes. With our limited amounts of $B$-band data this approach produces mostly 
Table 1. DIRECT ECLIPSing BinARIES in M31C

\begin{tabular}{|c|c|c|c|c|c|c|c|c|c|c|c|}
\hline $\begin{array}{c}\text { Name } \\
\text { (D31C) }\end{array}$ & $\begin{array}{c}\alpha_{J 2000.0} \\
\quad(\mathrm{deg})\end{array}$ & $\begin{array}{c}\delta_{J 2000.0} \\
(\mathrm{deg})\end{array}$ & $\begin{array}{c}P \\
(\text { days })\end{array}$ & $V_{\max }$ & $I_{\max }$ & $B_{\max }$ & $R_{1}$ & $R_{2}$ & $\begin{array}{c}i \\
(\operatorname{deg})\end{array}$ & $e$ & Comments \\
\hline V12262... & 11.1503 & 41.4888 & 2.0489 & 20.02 & 20.19 & 19.85 & 0.50 & 0.32 & 71 & 0.00 & \\
\hline V12594... & 11.1568 & 41.4962 & 2.3013 & 20.49 & 20.68 & 20.37 & 0.59 & 0.41 & 72 & 0.01 & V2763 D31B \\
\hline V10732... & 11.1246 & 41.3909 & 2.3048 & 20.65 & $\ldots$ & 20.34 & 0.42 & 0.34 & 84 & 0.00 & DEB \\
\hline V14662 ... & 11.2087 & 41.4686 & 2.8606 & 20.28 & 19.81 & 20.19 & 0.46 & 0.35 & 83 & 0.01 & DEB \\
\hline V10550... & 11.1219 & 41.3837 & 3.1687 & 19.35 & 19.26 & 19.18 & 0.50 & 0.49 & 90 & 0.00 & \\
\hline V12650... & 11.1582 & 41.4899 & 3.5500 & 19.19 & 18.96 & 19.13 & 0.50 & 0.49 & 89 & 0.02 & \\
\hline V14653... & 11.2081 & 41.4807 & 3.8839 & 20.60 & $\ldots$ & 20.36 & 0.50 & 0.49 & 90 & 0.02 & \\
\hline V14396... & 11.2035 & 41.3833 & 5.4260 & 21.32 & $\ldots$ & 21.80 & 0.63 & 0.37 & 90 & 0.00 & \\
\hline V9037 ... & 11.0969 & 41.4523 & 5.7735 & 19.22 & 19.13 & 19.24 & 0.31 & 0.26 & 76 & 0.14 & DEB \\
\hline V11295... & 11.1333 & 41.4223 & 7.6907 & 17.31 & 16.16 & 18.20 & 0.52 & 0.39 & 49 & 0.00 & W UMa \\
\hline V14439... & 11.2024 & 41.4577 & 9.1370 & 21.12 & 20.96 & 21.06 & 0.33 & 0.33 & 72 & 0.17 & DEB? \\
\hline V13944... & 11.1886 & 41.4667 & 11.5385 & 18.68 & 18.55 & 18.71 & 0.68 & 0.32 & 69 & 0.00 & Ma97 92 \\
\hline
\end{tabular}

Note. - V9037 D31C with period $P=5.7735$ days is a good detached eclipsing binary (DEB) candidate, with significant eccentricity. V12594 D31C was found in Paper I as V2763 D31B, with $P=2.302$ days, $V_{\max }=20.51$ and $I_{\max }=20.84$. 

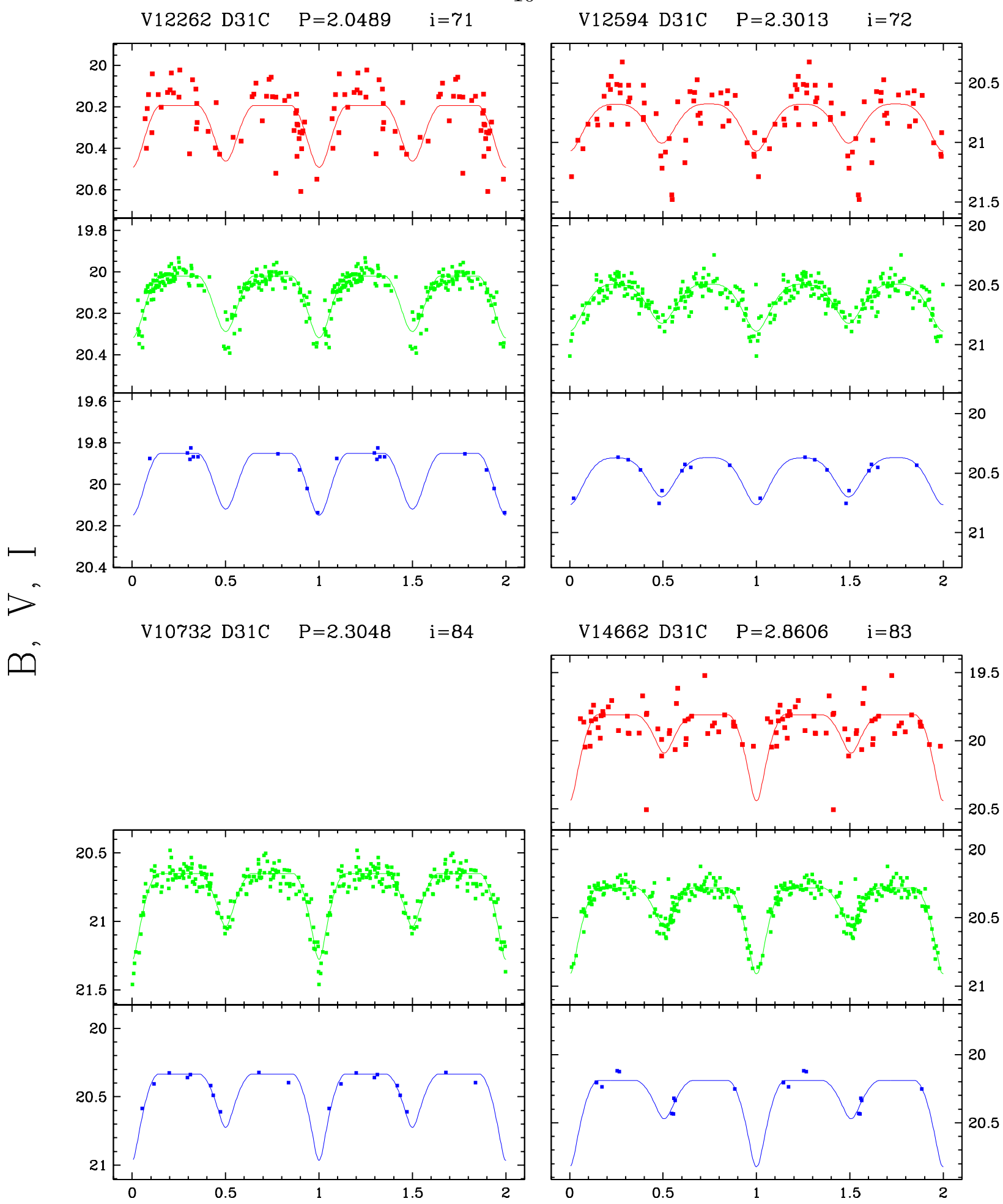

\section{Phase}

Fig. 3.- BVI lightcurves of eclipsing binaries found in the field M31C. The thin continuous line represents the best fit model for each star and photometric band. $B$-band lightcurve is shown in the bottom panel and $I$-band lightcurve (when present) is shown in the top panel. 

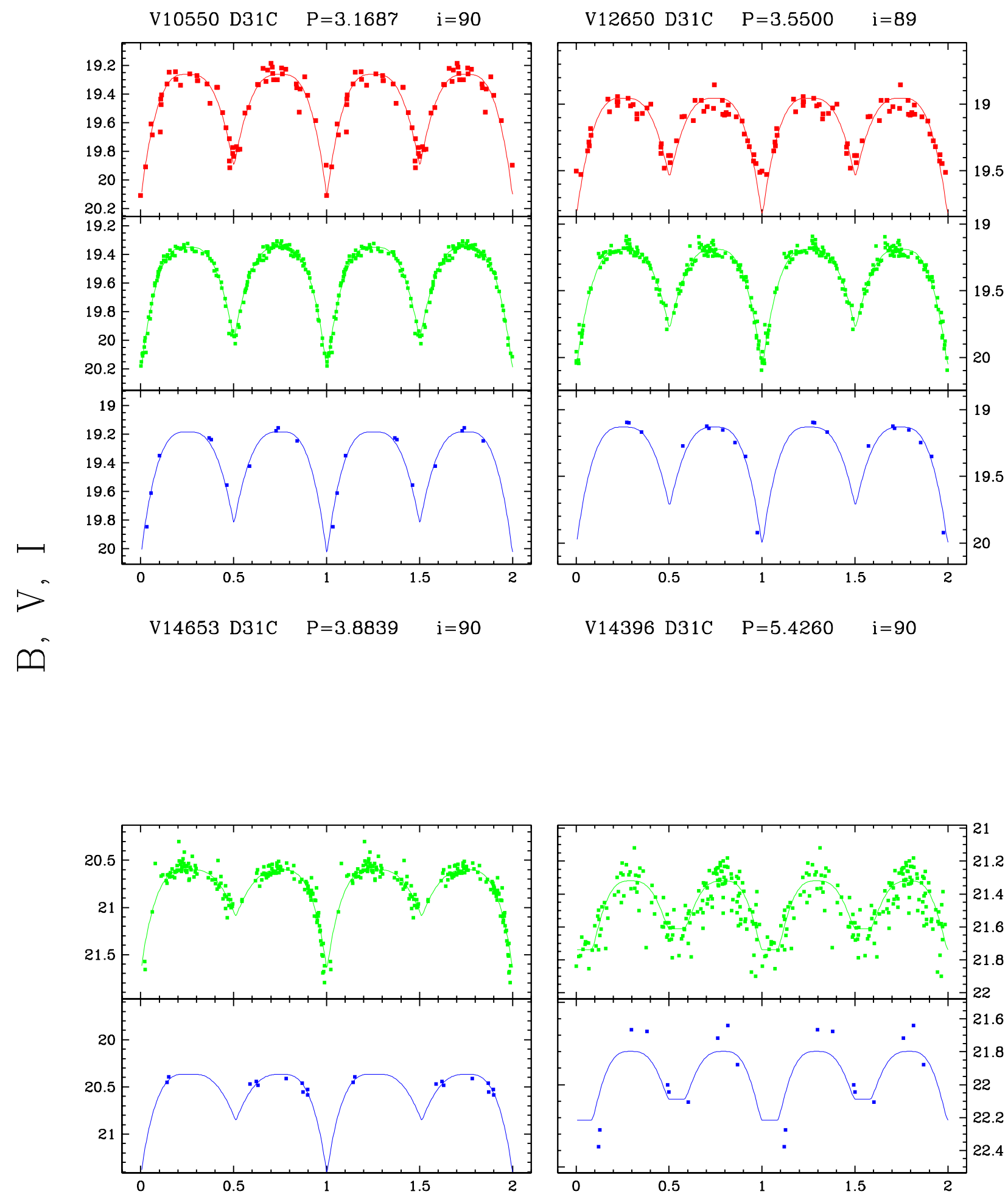

Phase

Fig. 3.- Continued. 


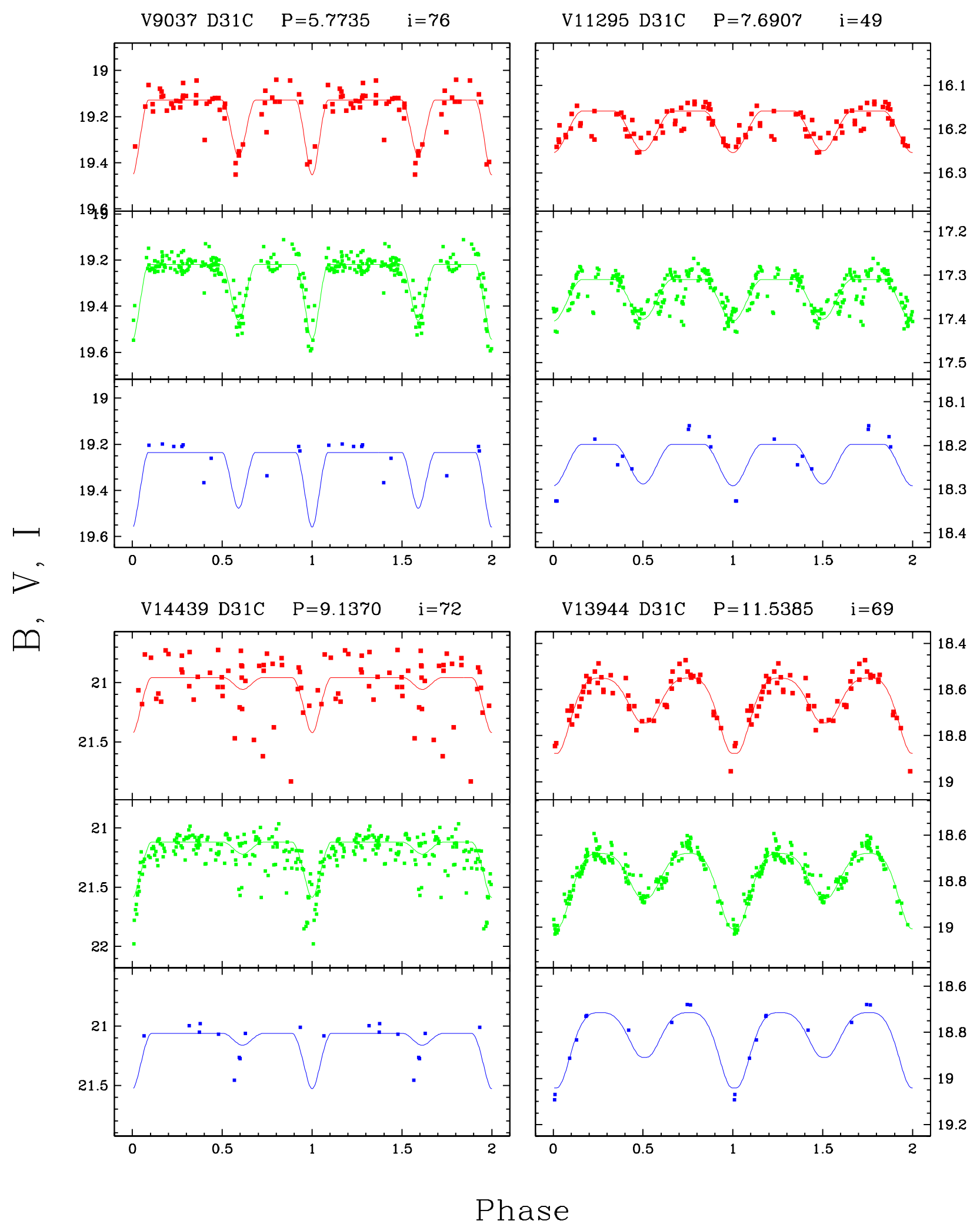

Fig. 3.- Continued. 


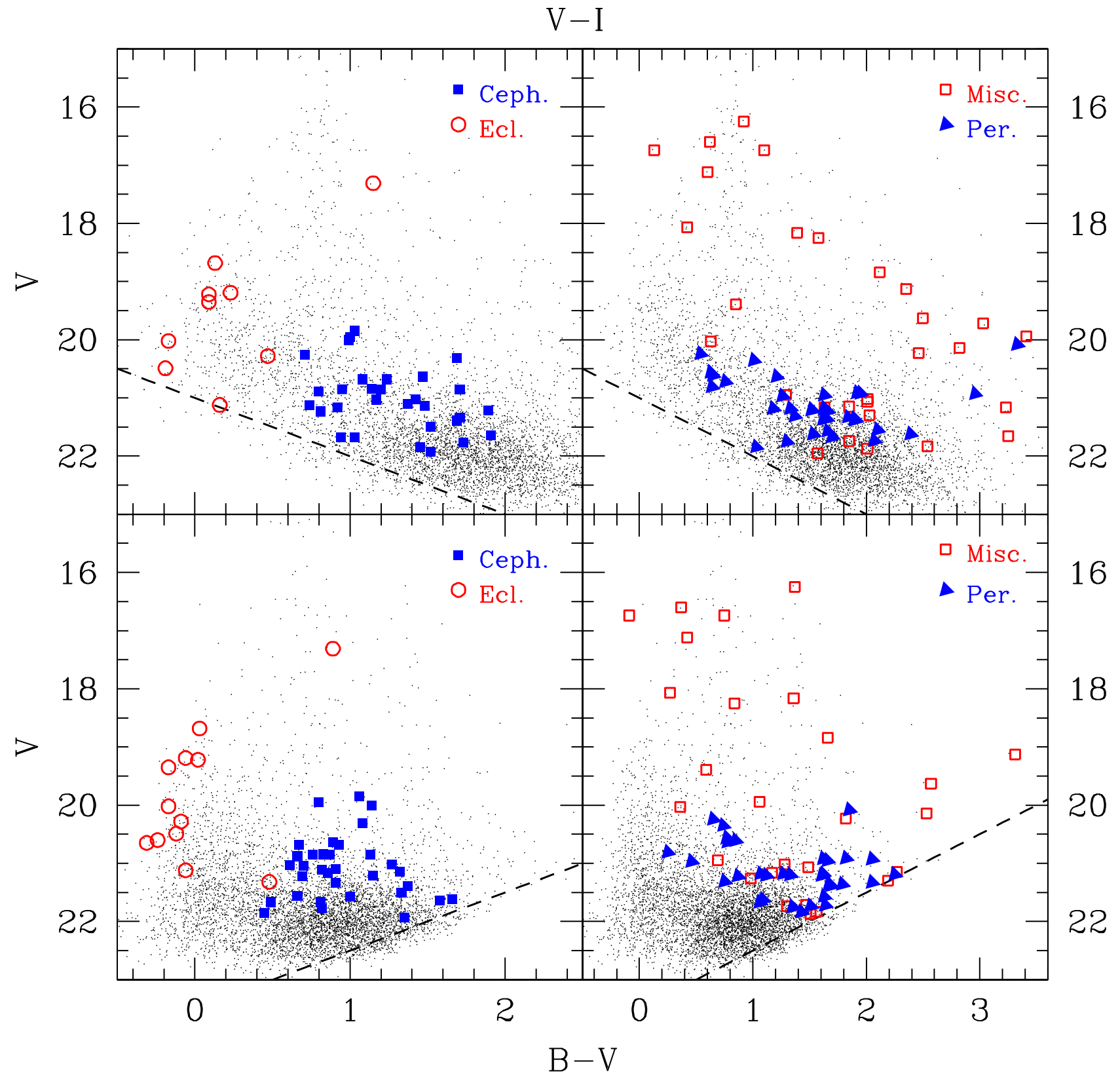

Fig. 4.- $V, V-I$ (upper panels) and $B, B-V$ (lower panels) color-magnitude diagrams for the variable stars found in the field M31C. The eclipsing binaries and Cepheids are plotted in the left panels and the other periodic variables and miscellaneous variables are plotted in the right panels. The dashed lines correspond to the $I$ detection limit of $I \sim 21 \mathrm{mag}$ (upper panels) and the $B$ detection limit of $B \sim 23.5 \mathrm{mag}$ (lower panels). 
satisfactory results, but extending the template-fitting approach of Stetson (1996) to the $B$-band (and possibly other popular bands) would be most useful.

\subsection{Other periodic variables}

For many of the variables preliminary classified as Cepheids we decided upon closer examination to classify them as "other periodic variables". In Table 3 we present the parameters of 37 possible periodic variables, other than Cepheids and eclipsing binaries, in the M31C field, sorted by the increasing period $P$. For each variable we present its name, J2000.0 coordinates, period $P$, error-weighted mean magnitudes $\bar{V}$ and (when available) $\bar{I}, \bar{B}$. To quantify the amplitude of the variability, we also give the standard deviations of the measurements in the $B V I$ bands, $\sigma_{V}, \sigma_{I}$ and $\sigma_{B}$.

Note that in most cases the periods were derived by fitting the template Cepheids lightcurves, so they should only be treated as the first approximation of the true period. Many of these

periodic variables are Type II Cepheids (W Virginis and RV Tauri variables), based on their light curves and their location on the P-L diagram (Figure 母).

\subsection{Miscellaneous variables}

In Table 1 we present the parameters of 31 miscellaneous variables in the M31C field, sorted by increasing value of the mean magnitude $\bar{V}$. For each variable we present its name, J2000.0 coordinates and mean magnitudes $\bar{V}, \bar{I}$ and $\bar{B}$. To quantify the amplitude of the variability, we also give the standard deviations of the measurements in VIB bands, $\sigma_{V}, \sigma_{I}$ and $\sigma_{B}$. In the "Comments" column we give a rather broad sub-classification of the variability: LP - possible long-period variable; Irr - irregular variable. In Figure $\mathbf{8}$ we show the unphased VI lightcurves of the miscellaneous variables.

Most of the miscellaneous variables seem to represent the LP type of variability, with few variables showing irregular variations. However, inspection of the color-magnitude diagram (Figure 4 ) reveals that many of the miscellaneous variables land in the CMD in the same area as the RV Tau variables, which suggests they are Type II Cepheids.

\subsection{Comparison with other catalogs}

The area of M31C field has not been observed frequently before and the only overlapping variable star catalog is given by Magnier et al. (1997, hereafter Ma97). Out of 14 variable stars in Ma97 which are located in our M31C field, we cross-identified 13. Of these 13 stars, four (Ma97 $79,84,88,91)$ we did not classify as variables $\left(J_{S}=0.72,-0.04,0.34,0.43\right)$. Of the remaining 

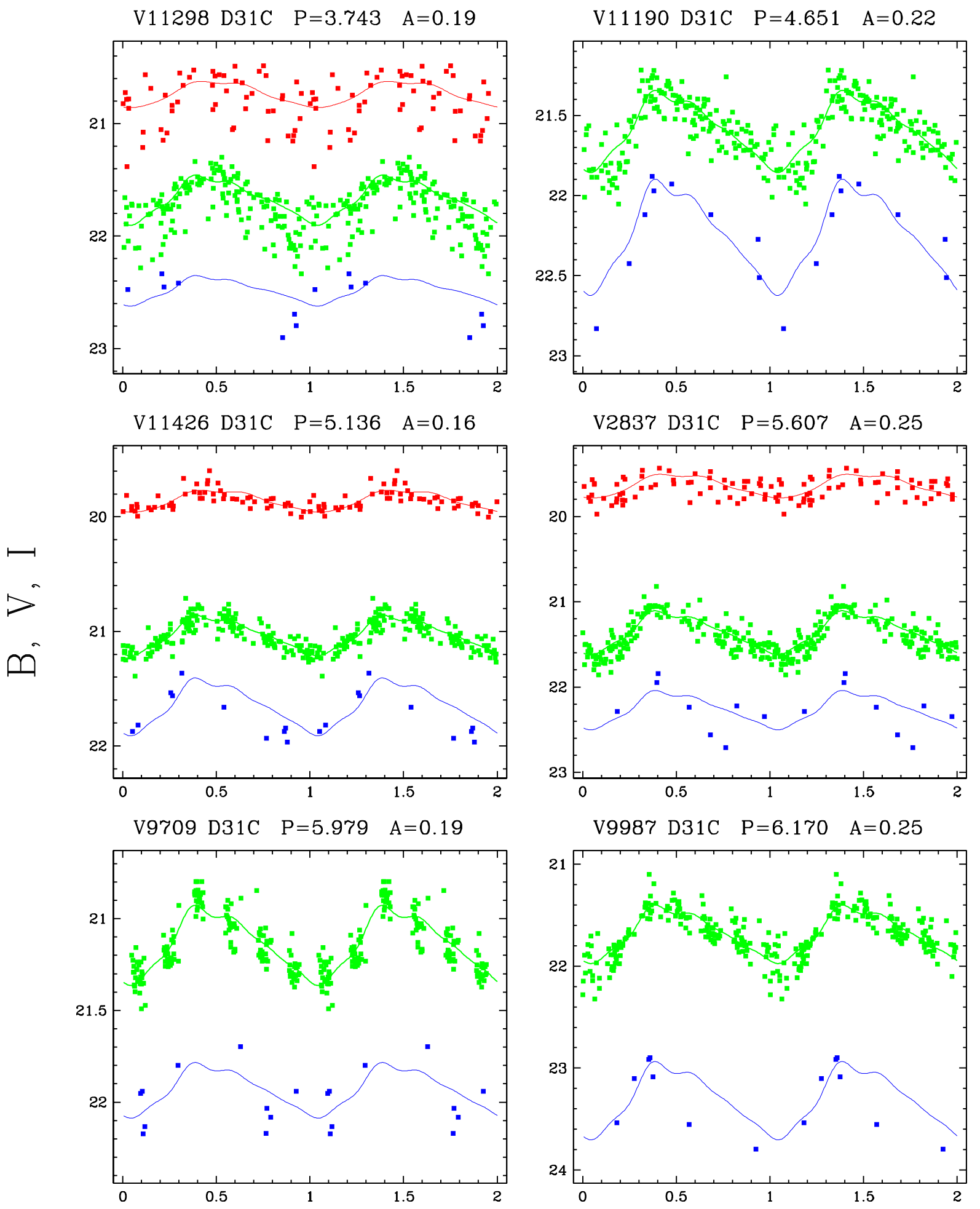

\section{Phase}

Fig. 5.- BVI lightcurves of Cepheid variables found in the field M31C. The thin continuous line represents the best fit Cepheid template for each star and photometric band. $B$ (if present) is always the faintest and $I$ (if present) is always the brightest. 
$-16-$
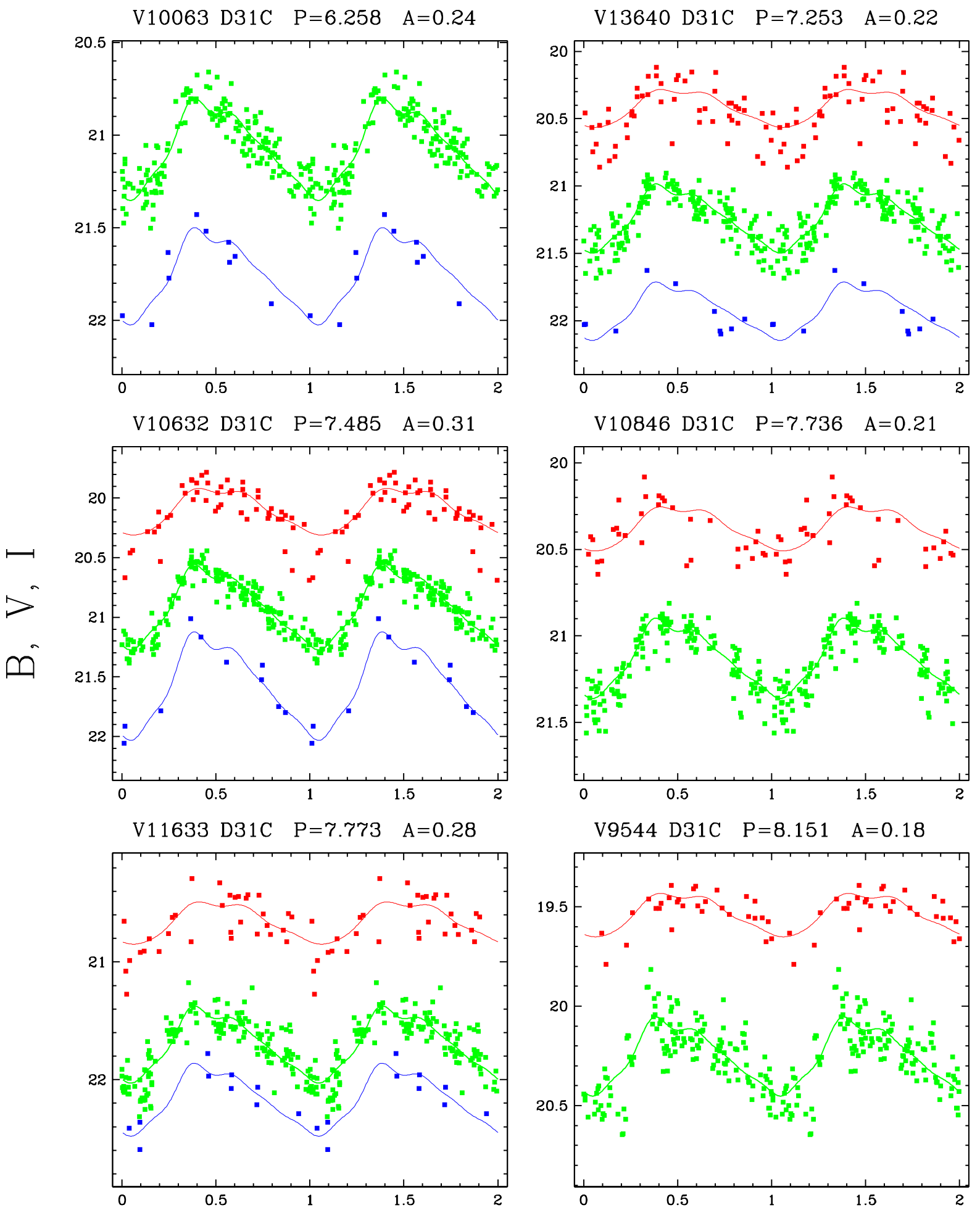

Phase

Fig. 5.- Continued. 

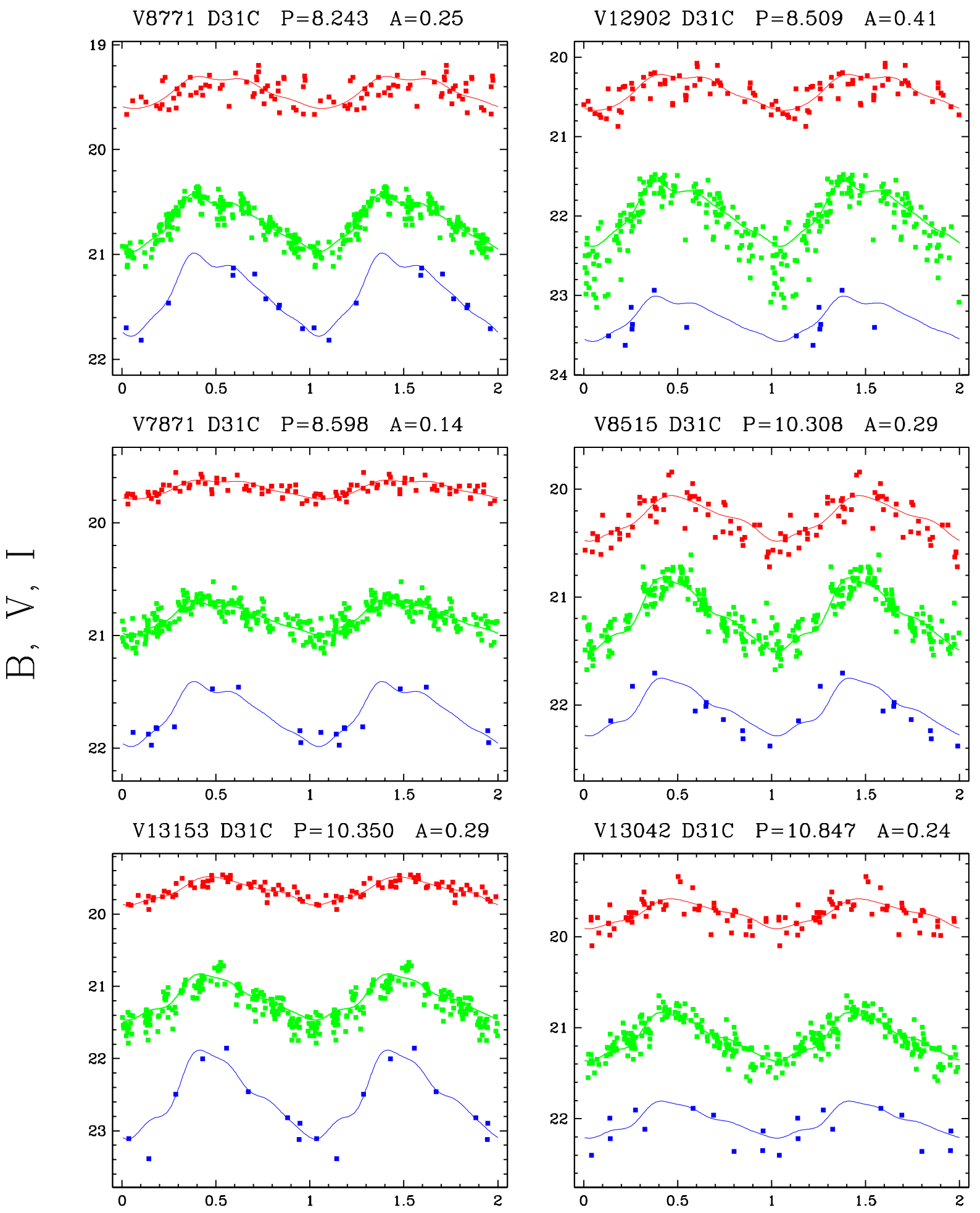

Phase

Fig. 5.- Continued. 
$-18-$
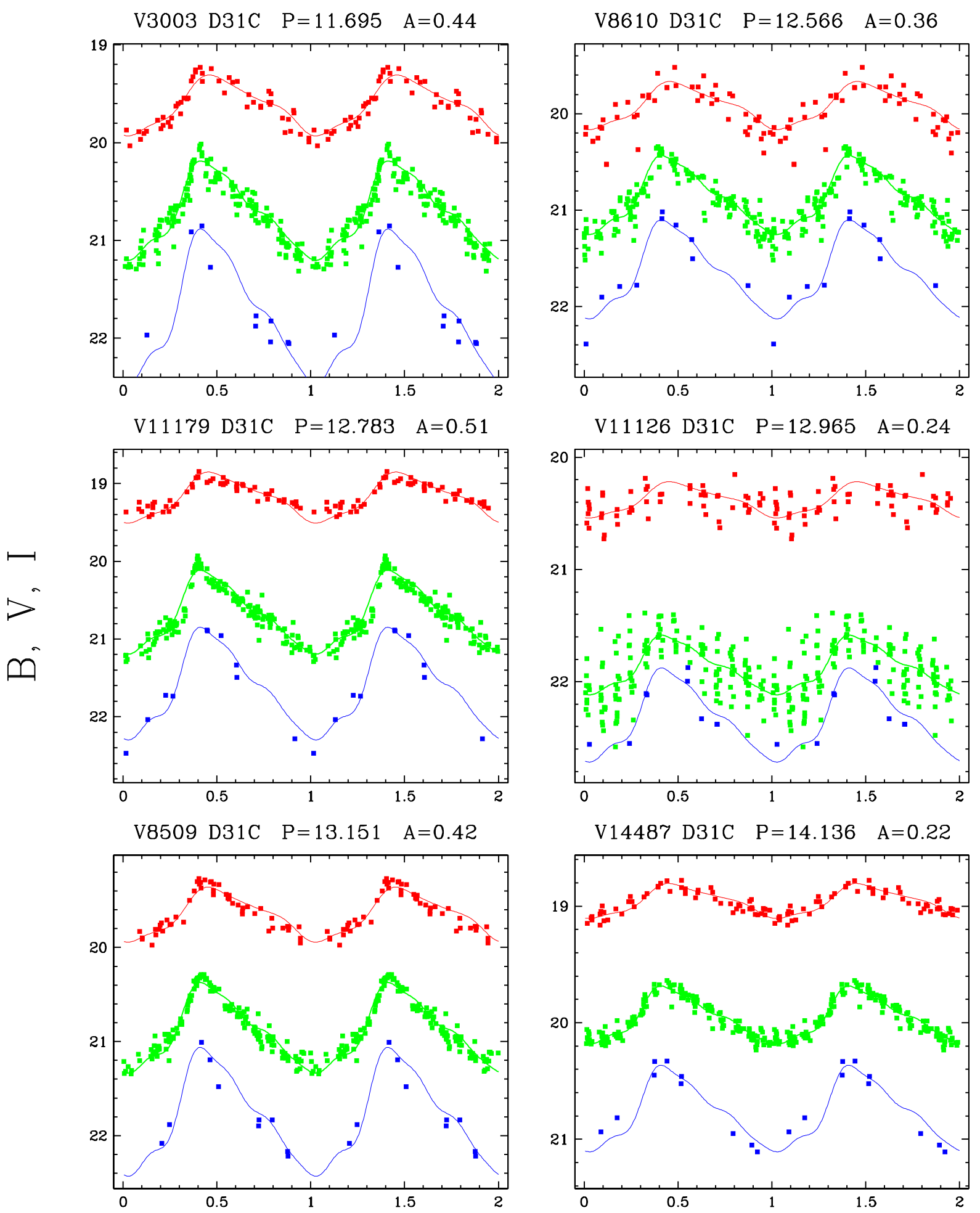

Phase

Fig. 5.- Continued. 
$-19-$
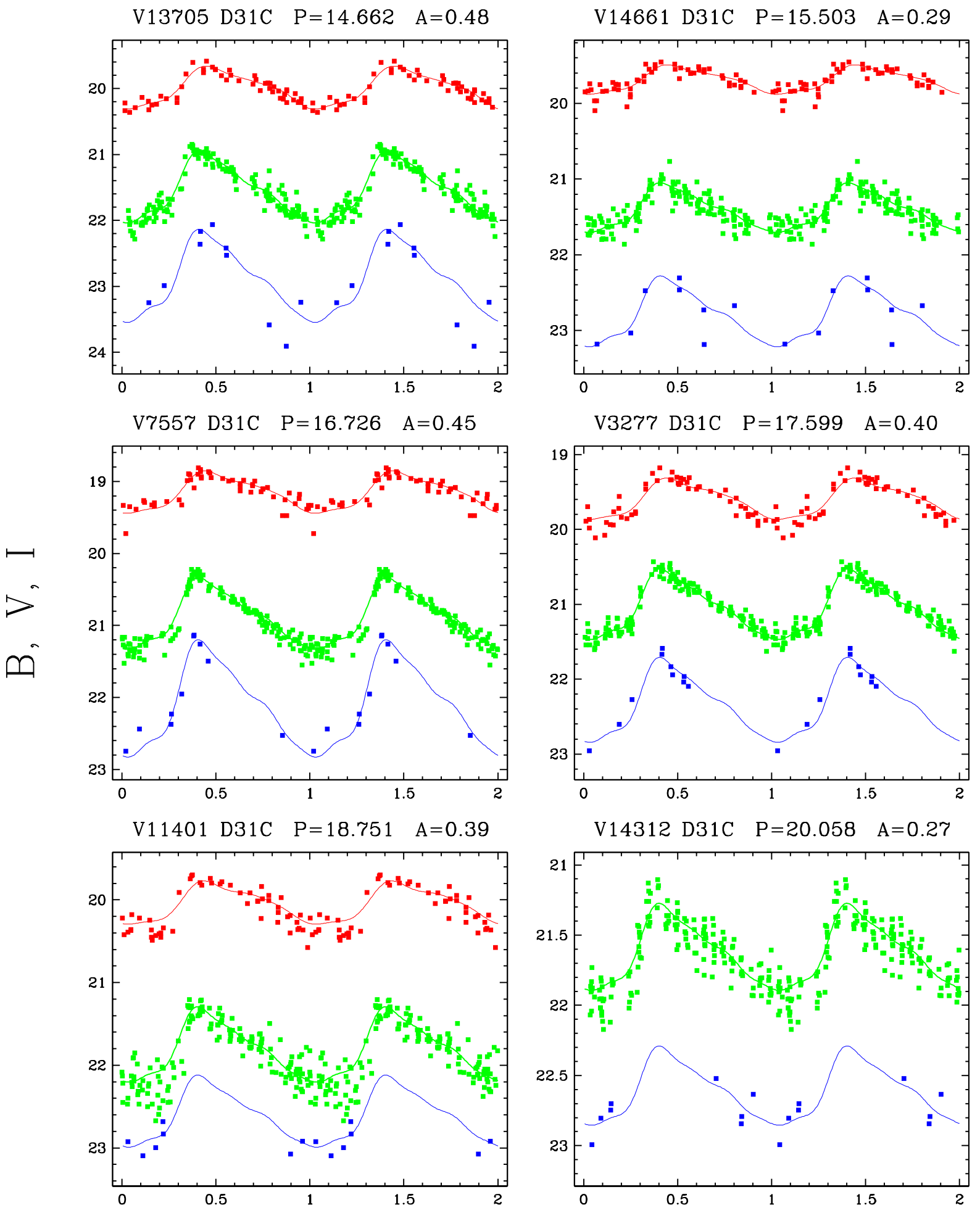

Phase

Fig. 5.- Continued. 

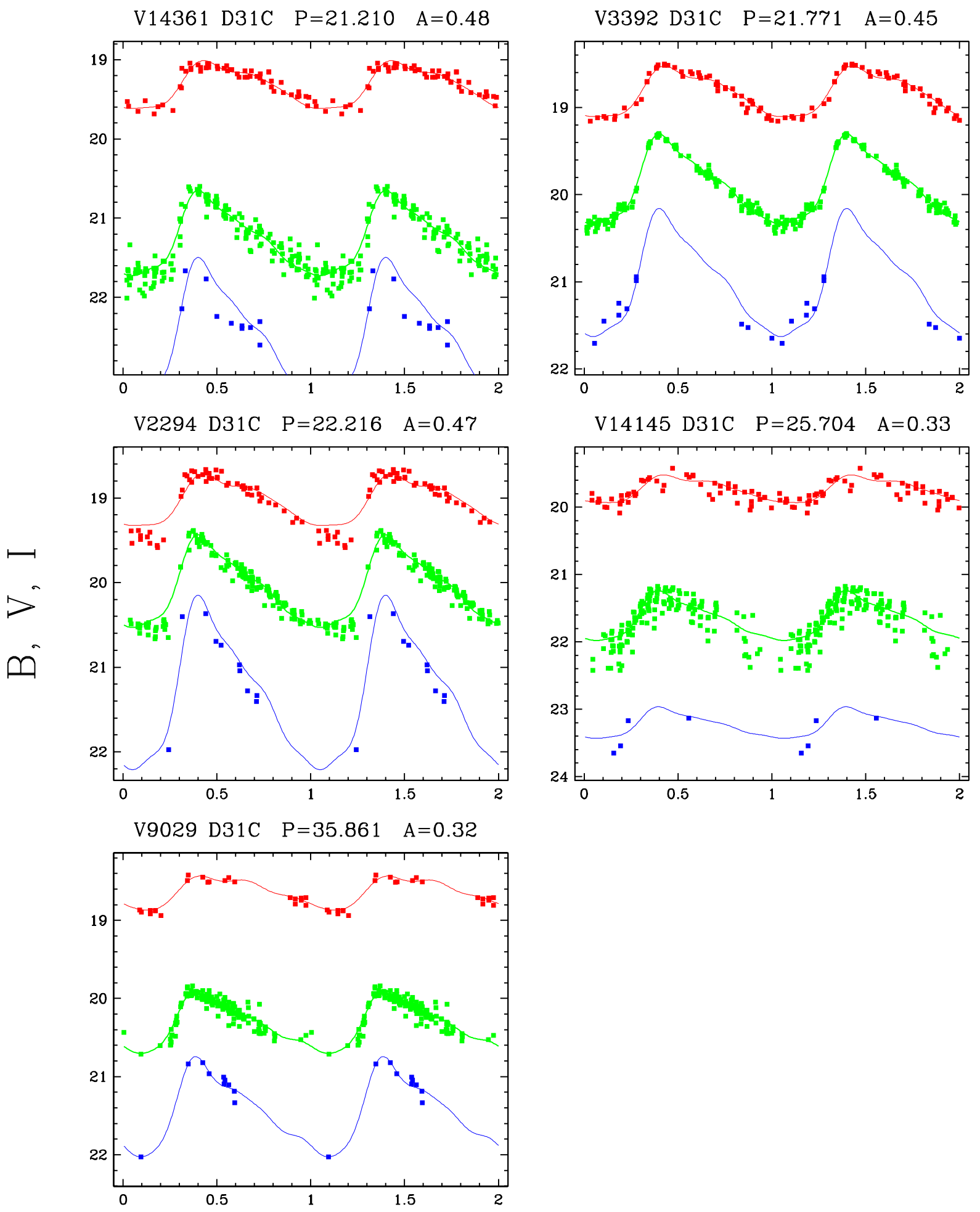

Phase

Fig. 5.- Continued. 

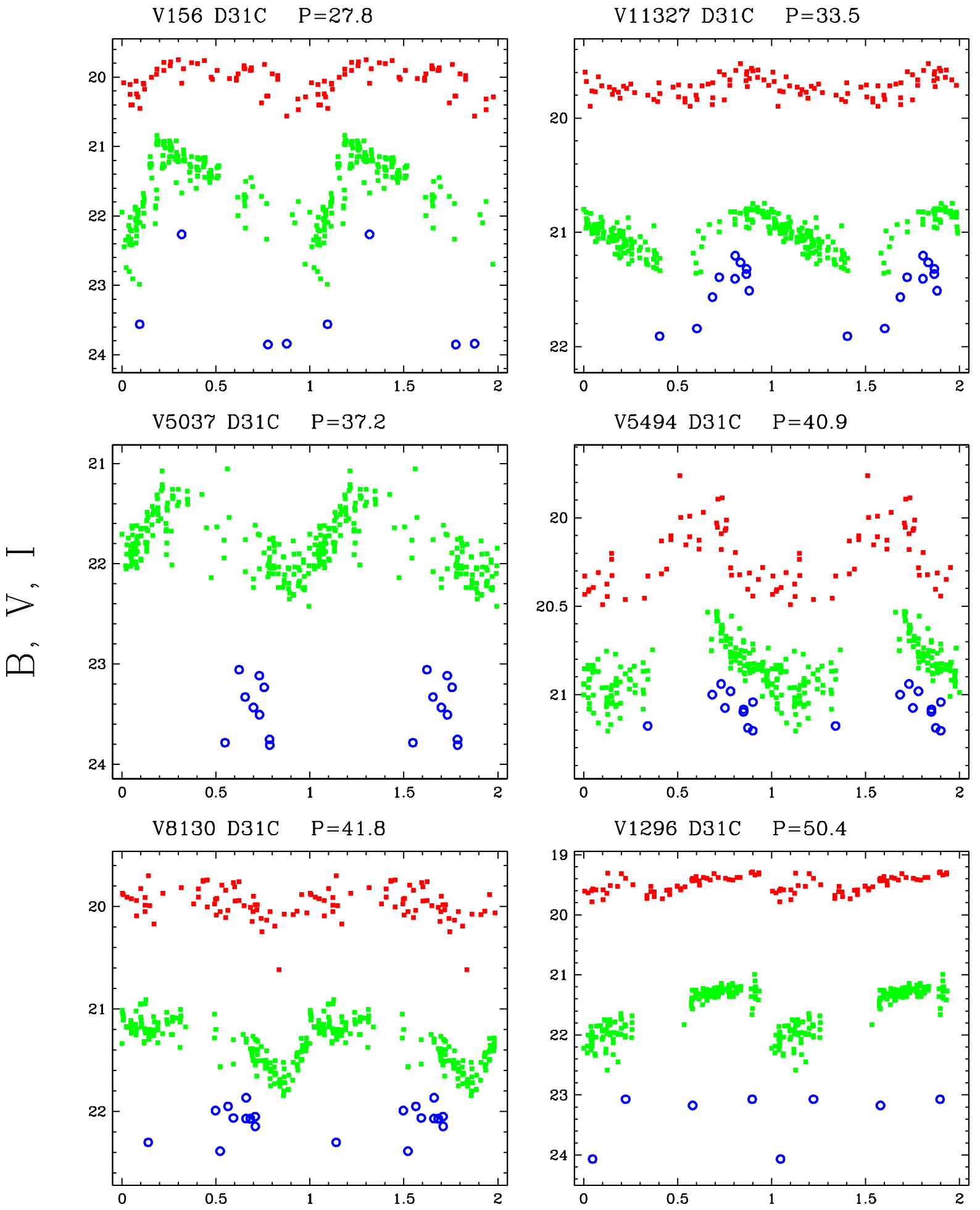

\section{Phase}

Fig. 6. - BVI lightcurves of other periodic variables found in the field M31C. B-band data (shown with the open circles, if present) is usually the faintest and $I$ (if present) is usually the brightest. 

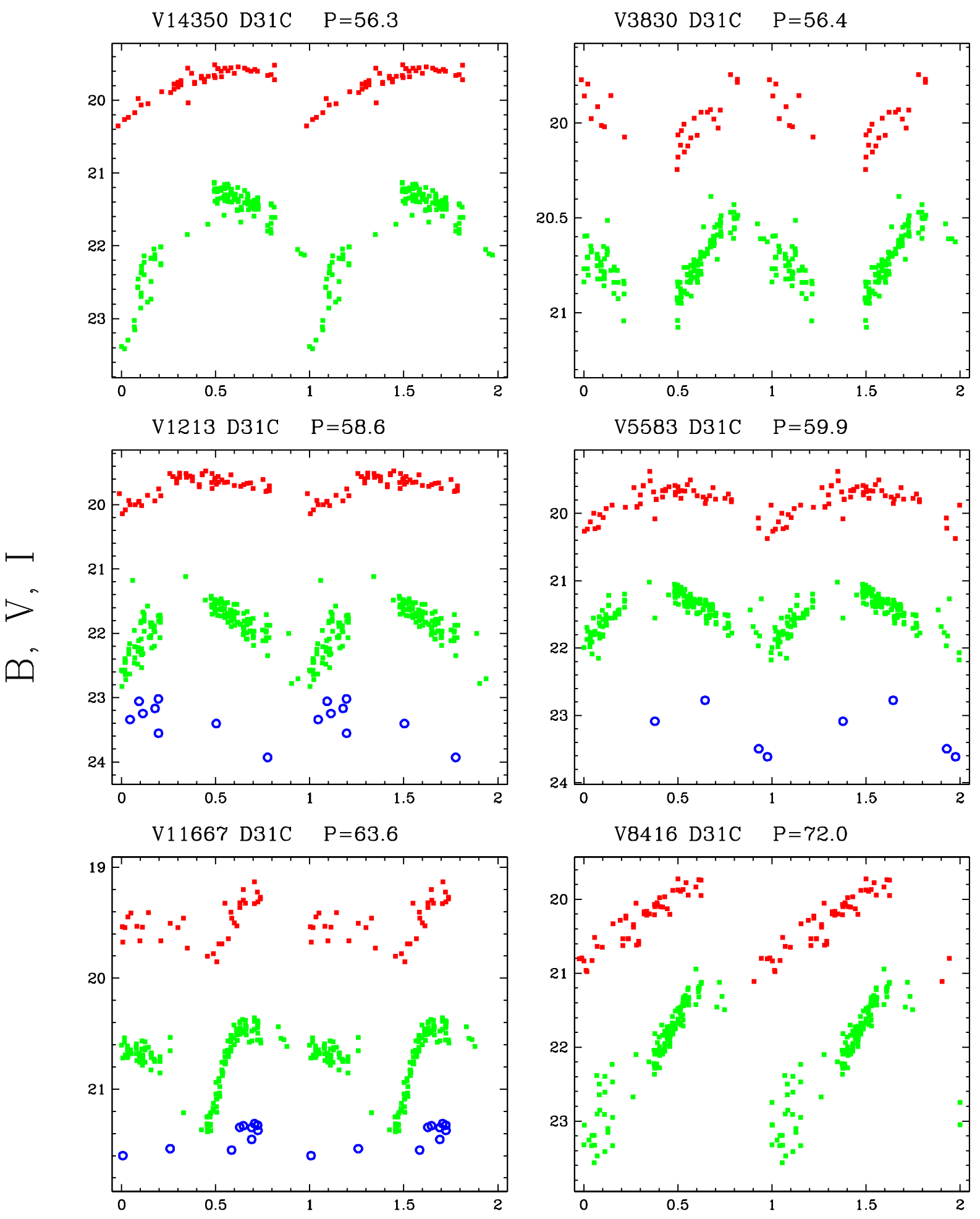

Phase

Fig. 6.- Continued. 

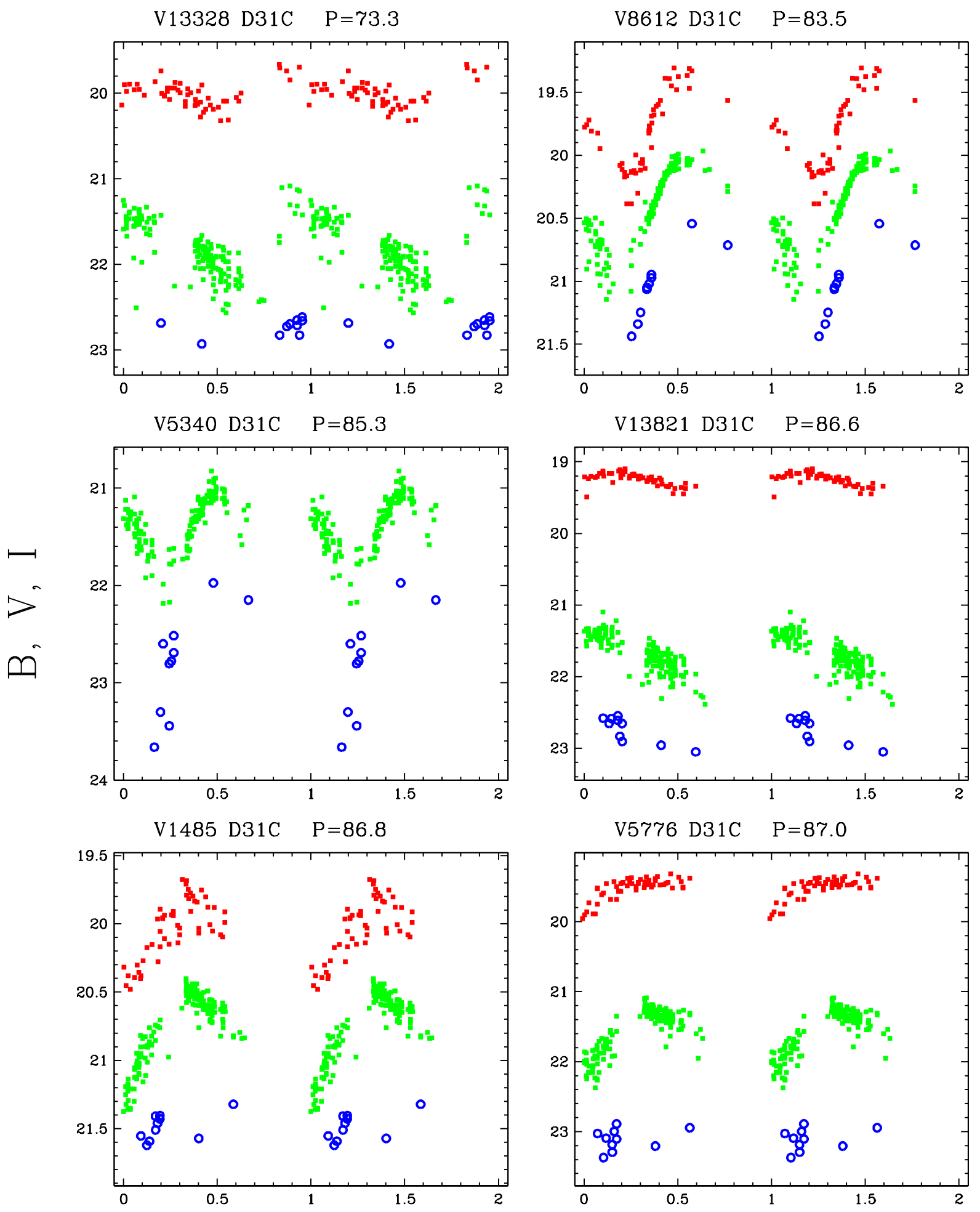

Phase

Fig. 6.- Continued. 

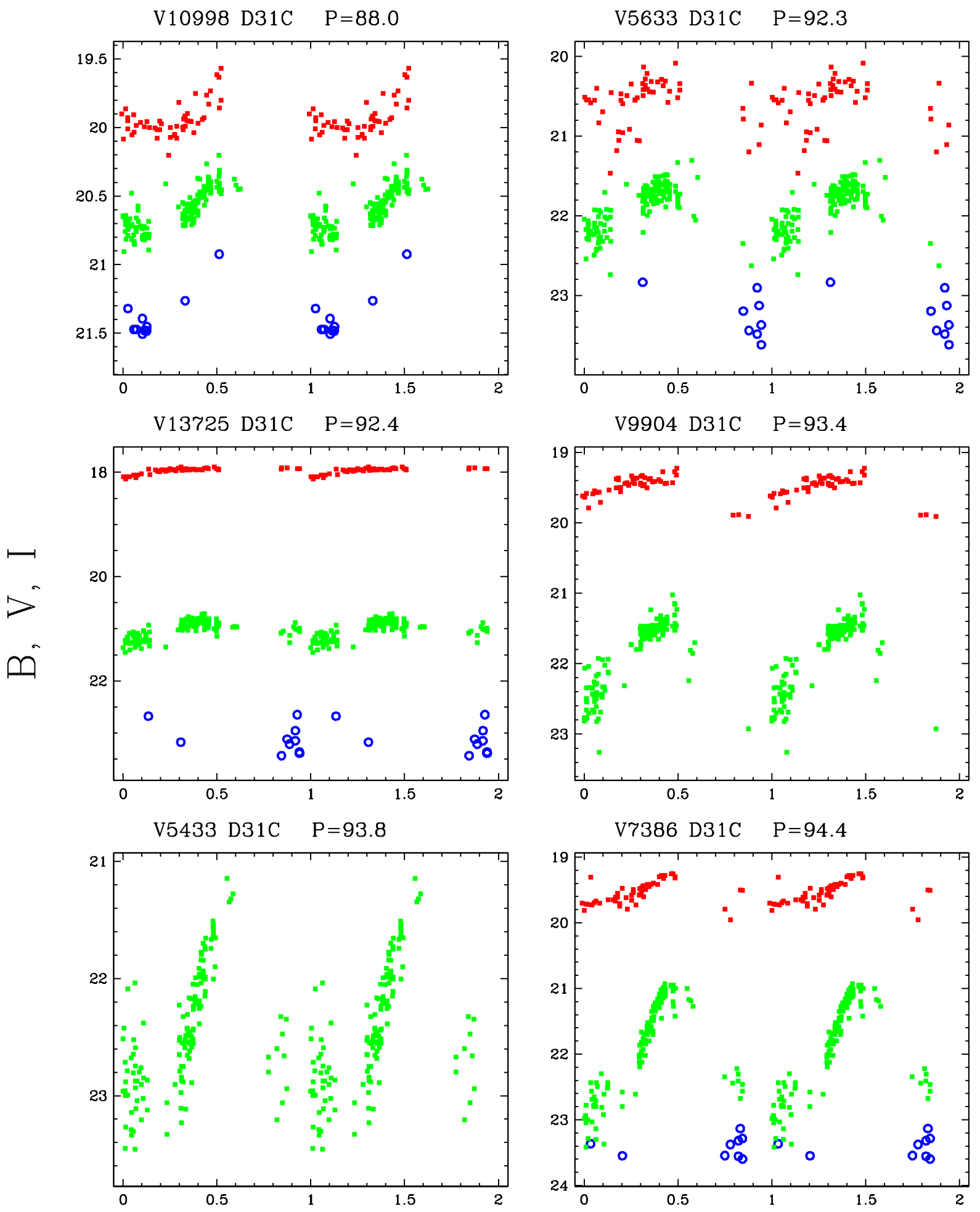

Phase

Fig. 6.- Continued. 

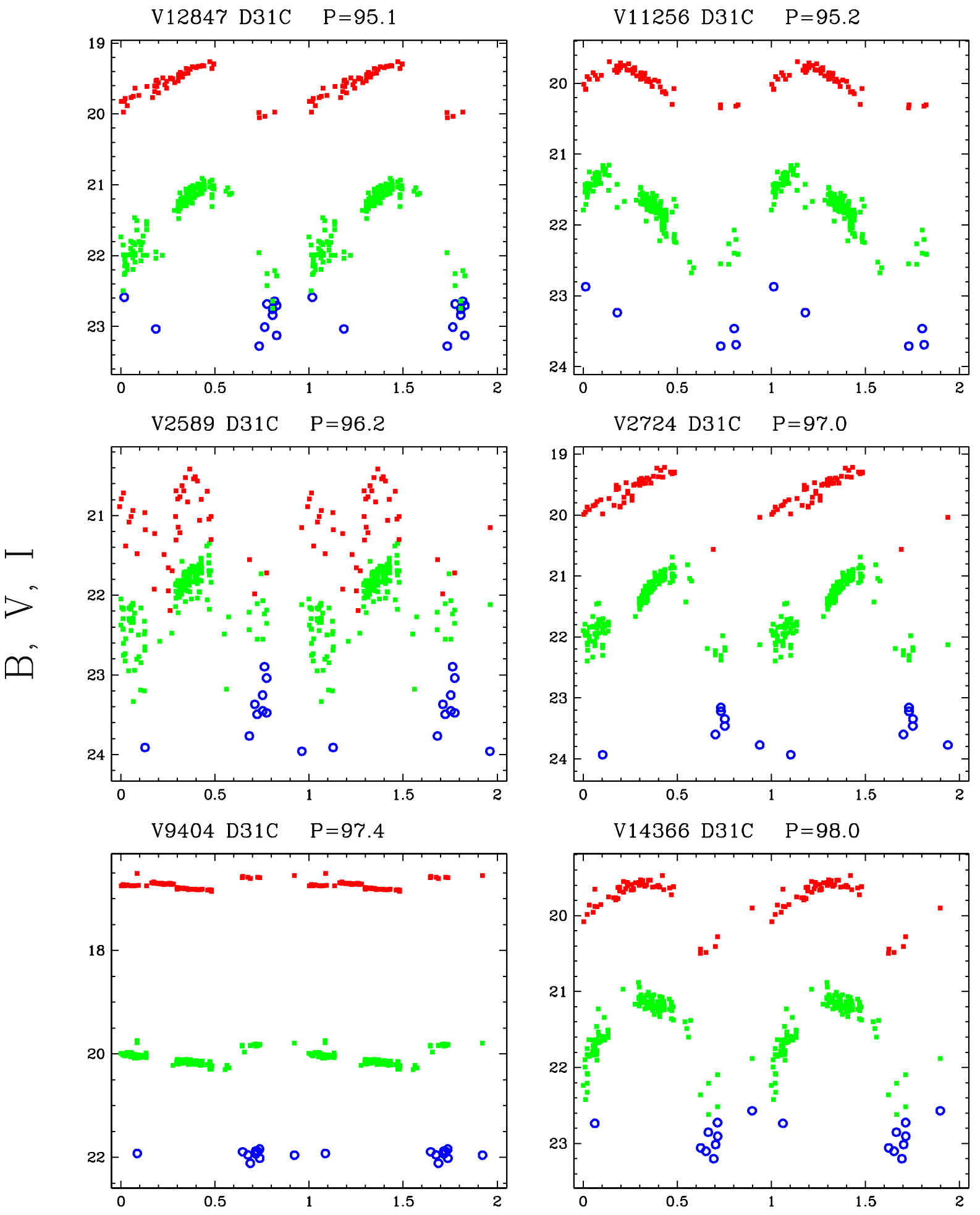

Phase

Fig. 6.- Continued. 

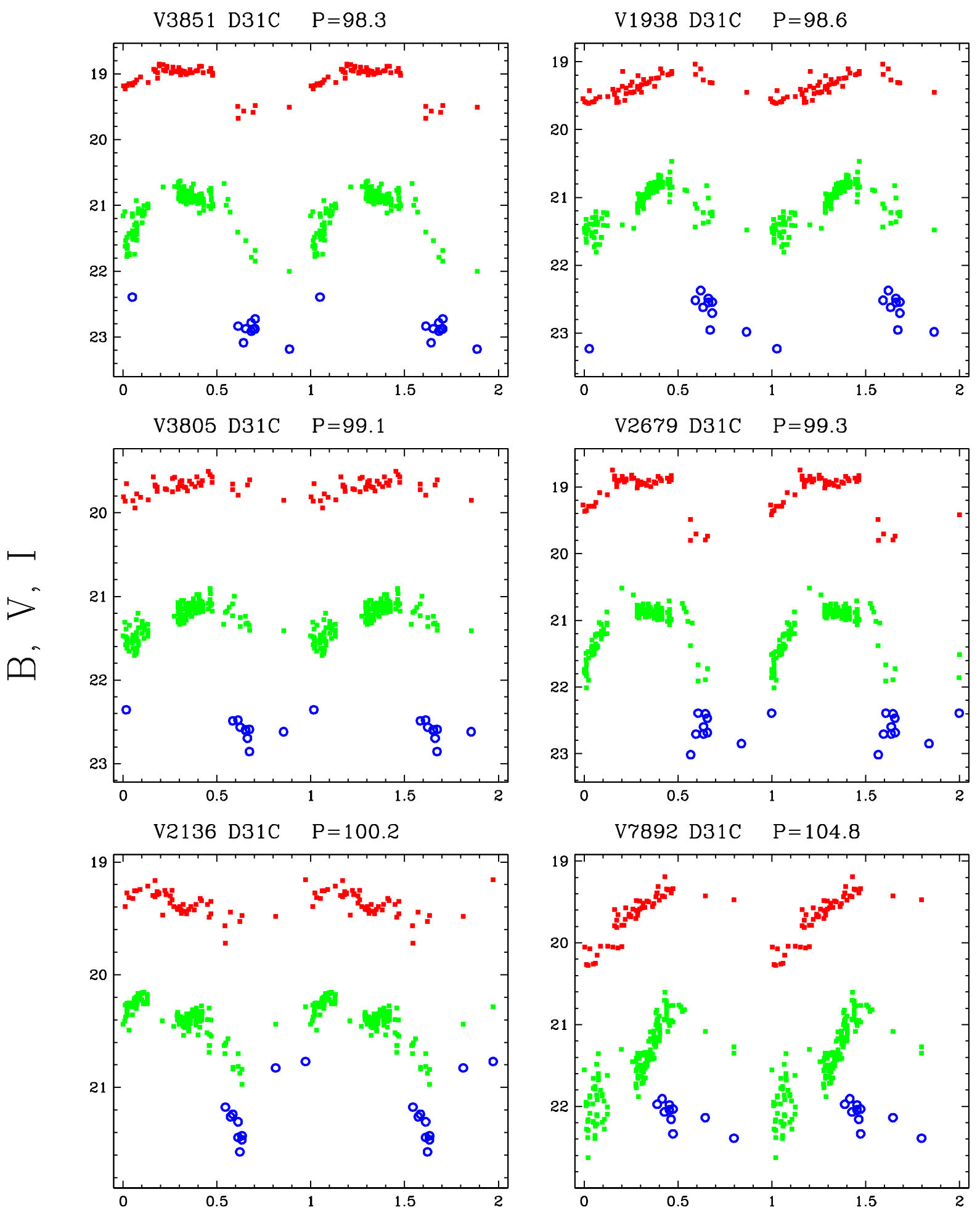

Phase

Fig. 6.- Continued. 
Table 2. DIRECT Cepheids in M31C

\begin{tabular}{|c|c|c|c|c|c|c|c|c|}
\hline $\begin{array}{c}\text { Name } \\
\text { (D31C) }\end{array}$ & $\begin{array}{c}\alpha_{J 2000.0} \\
(\mathrm{deg})\end{array}$ & $\begin{array}{c}\delta_{J 2000.0} \\
(\mathrm{deg})\end{array}$ & $\begin{array}{c}P \\
(\text { days })\end{array}$ & $\langle V\rangle$ & $\langle I\rangle$ & $\langle B\rangle$ & $A$ & Comments \\
\hline V11298... & 11.1346 & 41.3806 & 3.743 & 21.67 & 20.73 & 22.48 & 0.19 & \\
\hline V11190... & 11.1339 & 41.3424 & 4.651 & 21.56 & $\ldots$ & 22.22 & 0.22 & \\
\hline V11426... & 11.1363 & 41.4097 & 5.136 & 21.03 & 19.86 & 21.64 & 0.16 & Ma97 85 \\
\hline V2837 ... & 11.0159 & 41.4518 & 5.607 & 21.34 & 19.63 & 22.25 & 0.25 & \\
\hline V9709.... & 11.1081 & 41.4021 & 5.979 & 21.11 & $\ldots$ & 21.93 & 0.19 & \\
\hline V9987.... & 11.1141 & 41.3523 & 6.170 & 21.62 & $\ldots$ & 23.28 & 0.25 & \\
\hline V10063... & 11.1129 & 41.4347 & 6.258 & 21.04 & $\ldots$ & 21.74 & 0.24 & \\
\hline V13640... & 11.1831 & 41.4072 & 7.253 & 21.23 & 20.42 & 21.92 & 0.22 & \\
\hline V10632 ... & 11.1225 & 41.4122 & 7.485 & 20.88 & 20.08 & 21.54 & 0.31 & Ma97 83 \\
\hline V10846... & 11.1229 & 41.5087 & 7.736 & 21.12 & 20.38 & $\ldots$ & 0.21 & V1562 D31B \\
\hline V11633 ... & 11.1414 & 41.3671 & 7.773 & 21.67 & 20.64 & 22.16 & 0.28 & \\
\hline V9544 ... & 11.1021 & 41.5129 & 8.151 & 20.25 & 19.54 & $\ldots$ & 0.18 & V643 D31B \\
\hline V8771.... & 11.0909 & 41.4971 & 8.243 & 20.68 & 19.44 & 21.35 & 0.25 & V129 D31B \\
\hline V12902 ... & 11.1635 & 41.5022 & 8.509 & 21.93 & 20.41 & 23.28 & 0.41 & V2977 D31B \\
\hline V7871 ... & 11.0829 & 41.3443 & 8.598 & 20.84 & 19.70 & 21.67 & 0.14 & \\
\hline V8515... & 11.0915 & 41.3549 & 10.308 & 21.16 & 20.24 & 22.02 & 0.29 & \\
\hline V13153... & 11.1715 & 41.4072 & 10.350 & 21.14 & 19.66 & 22.46 & 0.29 & Ma97 90 \\
\hline V13042 ... & 11.1698 & 41.3994 & 10.847 & 21.10 & 19.73 & 22.01 & 0.24 & \\
\hline V3003 .... & 11.0171 & 41.4620 & 11.695 & 20.68 & 19.60 & 21.61 & 0.44 & \\
\hline V8610.... & 11.0916 & 41.3966 & 12.566 & 20.85 & 19.90 & 21.61 & 0.36 & Ma97 77 \\
\hline V11179... & 11.1317 & 41.4136 & 12.783 & 20.64 & 19.17 & 21.53 & 0.51 & \\
\hline V11126... & 11.1299 & 41.4356 & 12.965 & 21.85 & 20.40 & 22.30 & 0.24 & \\
\hline V8509.... & 11.0909 & 41.3723 & 13.151 & 20.85 & 19.65 & 21.72 & 0.42 & Ma97 76 \\
\hline V14487... & 11.2027 & 41.4876 & 14.136 & 19.95 & 18.95 & 20.75 & 0.22 & Ma97 95 \\
\hline V13705... & 11.1822 & 41.4766 & 14.662 & 21.50 & 19.98 & 22.83 & 0.48 & \\
\hline V14661... & 11.2092 & 41.4527 & 15.503 & 21.39 & 19.70 & 22.76 & 0.29 & \\
\hline V7557 ... & 11.0783 & 41.3467 & 16.726 & 20.85 & 19.14 & 21.98 & 0.45 & Ma97 75 \\
\hline V3277 ... & 11.0238 & 41.3485 & 17.599 & 21.02 & 19.60 & 22.29 & 0.40 & \\
\hline V11401... & 11.1358 & 41.4061 & 18.751 & 21.77 & 20.04 & 22.59 & 0.39 & \\
\hline V14312 ... & 11.2015 & 41.3861 & 20.058 & 21.57 & $\ldots$ & 22.57 & 0.27 & \\
\hline V14361... & 11.2006 & 41.4493 & 21.210 & 21.21 & 19.32 & 22.36 & 0.48 & Ma97 94 \\
\hline V3392 .... & 11.0229 & 41.4243 & 21.771 & 19.85 & 18.82 & 20.91 & 0.45 & \\
\hline V2294... & 11.0078 & 41.5020 & 22.216 & 20.01 & 19.02 & 21.15 & 0.47 & \\
\hline V14145... & 11.1950 & 41.4465 & 25.704 & 21.64 & 19.73 & 23.22 & 0.33 & \\
\hline V9029 ... & 11.0996 & 41.3533 & 35.861 & 20.31 & 18.62 & 21.39 & 0.32 & \\
\hline
\end{tabular}

Note. - V10846 D31C was found in Paper I as V1562 D31B, with $P=7.784$ days, $\langle V\rangle=21.20$ and $\langle I\rangle=20.43$; V9544 D31C was found as V643 D31B, with $P=7.889$ days, $\langle V\rangle=20.39$ and $\langle I\rangle=19.52$; V8771 D31C was found as V129 D31B, with $P=8.242$ days, $\langle V\rangle=20.74$ and $\langle I\rangle=19.58$; V12902 D31C was found as V2977 D31B, with $P=8.518$ days, $\langle V\rangle=21.80$ and $\langle I\rangle=20.40$. 
Table 3. DIRECT Other Periodic Variables in M31C

\begin{tabular}{|c|c|c|c|c|c|c|c|c|c|c|}
\hline $\begin{array}{l}\text { Name } \\
\text { (D31C) }\end{array}$ & $\begin{array}{c}\alpha_{J 2000.0} \\
(\mathrm{deg})\end{array}$ & $\begin{array}{c}\delta_{J 2000.0} \\
(\mathrm{deg})\end{array}$ & $\begin{array}{c}P \\
(\text { days })\end{array}$ & $\bar{V}$ & $\bar{I}$ & $\bar{B}$ & $\sigma_{V}$ & $\sigma_{I}$ & $\sigma_{B}$ & Comments \\
\hline V156.... & 10.9877 & 41.3845 & 27.8 & 21.18 & 20.00 & 22.45 & 0.49 & 0.22 & 0.76 & RV Tau \\
\hline V11327... & 11.1349 & 41.3930 & 33.5 & 20.97 & 19.71 & 21.43 & 0.15 & 0.09 & 0.24 & \\
\hline V5037... & 11.0424 & 41.4547 & 37.2 & 21.72 & $\ldots$ & 23.36 & 0.30 & $\ldots$ & 0.29 & \\
\hline V5494 ... & 11.0515 & 41.3655 & 40.9 & 20.81 & 20.17 & 21.06 & 0.15 & 0.18 & 0.09 & \\
\hline V8130.... & 11.0829 & 41.4698 & 41.8 & 21.31 & 19.94 & 22.06 & 0.22 & 0.15 & 0.16 & \\
\hline V1296 .... & 11.0011 & 41.3400 & 50.4 & 21.36 & 19.46 & 23.15 & 0.37 & 0.13 & 0.48 & \\
\hline V14350... & 11.2033 & 41.3398 & 56.3 & 21.36 & 19.71 & $\ldots$ & 0.55 & 0.21 & $\ldots$ & \\
\hline V3830.... & 11.0256 & 41.5107 & 56.4 & 20.72 & 19.96 & $\ldots$ & 0.14 & 0.13 & $\ldots$ & \\
\hline V1213.... & 10.9989 & 41.3831 & 58.6 & 21.73 & 19.66 & 23.24 & 0.33 & 0.17 & 0.30 & \\
\hline V5583... & 11.0529 & 41.3526 & 59.9 & 21.37 & 19.75 & 23.04 & 0.24 & 0.22 & 0.39 & \\
\hline V11667... & 11.1391 & 41.4679 & 63.6 & 20.63 & 19.42 & 21.41 & 0.27 & 0.18 & 0.11 & RV Tau \\
\hline V8416.... & 11.0869 & 41.4684 & 72.0 & 21.62 & 20.09 & $\ldots$ & 0.62 & 0.37 & $\ldots$ & \\
\hline V13328 ... & 11.1725 & 41.4973 & 73.3 & 21.67 & 19.97 & 22.73 & 0.33 & 0.15 & 0.10 & \\
\hline V8612.... & 11.0922 & 41.3798 & 83.5 & 20.24 & 19.70 & 20.89 & 0.29 & 0.31 & 0.27 & RV Tau \\
\hline V5340.... & 11.0475 & 41.4341 & 85.3 & 21.21 & $\ldots$ & 22.33 & 0.25 & $\ldots$ & 0.54 & \\
\hline V13821... & 11.1879 & 41.3777 & 86.6 & 21.62 & 19.23 & 22.71 & 0.25 & 0.09 & 0.18 & \\
\hline V1485.... & 11.0030 & 41.3578 & 86.8 & 20.61 & 19.96 & 21.46 & 0.25 & 0.22 & 0.10 & \\
\hline V5776 ... & 11.0518 & 41.4741 & 87.0 & 21.38 & 19.48 & 23.07 & 0.32 & 0.15 & 0.15 & \\
\hline V10998... & 11.1257 & 41.4913 & 88.0 & 20.56 & 19.93 & 21.33 & 0.15 & 0.12 & 0.18 & \\
\hline V5633 ... & 11.0524 & 41.3904 & 92.3 & 21.75 & 20.45 & 23.10 & 0.28 & 0.30 & 0.28 & \\
\hline V13725 ... & 11.1842 & 41.4296 & 92.4 & 20.93 & 17.97 & 22.98 & 0.17 & 0.05 & 0.28 & $\mathrm{LP}$ \\
\hline V9904 .... & 11.1114 & 41.3963 & 93.4 & 21.55 & 19.45 & $\ldots$ & 0.48 & 0.15 & $\ldots$ & \\
\hline V5433 ... & 11.0498 & 41.3979 & 93.8 & 22.10 & $\ldots$ & $\ldots$ & 0.51 & $\ldots$ & $\ldots$ & \\
\hline V7386 ... & 11.0716 & 41.4897 & 94.4 & 21.33 & 19.49 & 23.38 & 0.69 & 0.17 & 0.16 & \\
\hline V12847 ... & 11.1635 & 41.4683 & 95.1 & 21.18 & 19.55 & 22.80 & 0.45 & 0.21 & 0.23 & \\
\hline V11256... & 11.1332 & 41.4051 & 95.2 & 21.57 & 19.91 & 23.20 & 0.32 & 0.17 & 0.35 & \\
\hline V2589.... & 11.0152 & 41.3759 & 96.2 & 21.84 & 20.81 & 23.27 & 0.39 & 0.45 & 0.35 & \\
\hline V2724... & 11.0175 & 41.3498 & 97.0 & 21.19 & 19.55 & 23.44 & 0.44 & 0.26 & 0.29 & \\
\hline V9404 ... & 11.1038 & 41.3895 & 97.4 & 20.08 & 16.75 & 21.93 & 0.11 & 0.08 & 0.08 & LP \\
\hline V14366 ... & 11.2033 & 41.3553 & 98.0 & 21.21 & 19.69 & 22.82 & 0.36 & 0.26 & 0.21 & \\
\hline V3851... & 11.0285 & 41.4277 & 98.3 & 20.91 & 18.99 & 22.73 & 0.30 & 0.20 & 0.21 & \\
\hline V1938.... & 11.0045 & 41.4926 & 98.6 & 20.95 & 19.32 & 22.61 & 0.28 & 0.15 & 0.27 & \\
\hline V3805 ... & 11.0294 & 41.3836 & 99.1 & 21.20 & 19.68 & 22.53 & 0.18 & 0.10 & 0.13 & \\
\hline V2679... & 11.0174 & 41.3357 & 99.3 & 20.92 & 18.97 & 22.54 & 0.32 & 0.27 & 0.21 & \\
\hline V2136.... & 11.0081 & 41.4458 & 100.2 & 20.35 & 19.34 & 21.09 & 0.15 & 0.11 & 0.27 & \\
\hline V7892 ... & 11.0818 & 41.3915 & 104.8 & 21.22 & 19.56 & 22.09 & 0.44 & 0.28 & 0.16 & \\
\hline V8038.... & 11.0851 & 41.3495 & 121.6 & 21.18 & 19.85 & 22.25 & 0.19 & 0.16 & 0.33 & \\
\hline
\end{tabular}


Table 4. DIRECT Miscellaneous Variables in M31C

\begin{tabular}{|c|c|c|c|c|c|c|c|c|c|}
\hline $\begin{array}{l}\text { Name } \\
\text { (D31C) }\end{array}$ & $\begin{array}{c}\alpha_{J 2000.0} \\
(\mathrm{deg})\end{array}$ & $\begin{array}{c}\delta_{J 2000.0} \\
(\mathrm{deg})\end{array}$ & $\bar{V}$ & $\bar{I}$ & $\bar{B}$ & $\sigma_{V}$ & $\sigma_{I}$ & $\sigma_{B}$ & Comments \\
\hline V5497 ... & 11.0489 & 41.4535 & 16.25 & 15.33 & 17.62 & 0.07 & 0.05 & 0.02 & \\
\hline V9306.... & 11.1039 & 41.3451 & 16.60 & 15.98 & 16.97 & 0.04 & 0.03 & 0.02 & \\
\hline V11839... & 11.1443 & 41.4177 & 16.74 & 16.61 & 16.65 & 0.04 & 0.03 & 0.01 & \\
\hline V9102 ... & 11.1007 & 41.3543 & 16.74 & 15.64 & 17.49 & 0.04 & 0.03 & 0.04 & \\
\hline V12381... & 11.1542 & 41.4424 & 17.12 & 16.52 & 17.54 & 0.04 & 0.02 & 0.03 & \\
\hline V13814... & 11.1854 & 41.4551 & 18.07 & 17.65 & 18.34 & 0.05 & 0.03 & 0.03 & \\
\hline V13102... & 11.1691 & 41.4512 & 18.16 & 16.77 & 19.52 & 0.07 & 0.03 & 0.04 & \\
\hline V13833... & 11.1854 & 41.4678 & 18.25 & 16.67 & 19.09 & 0.05 & 0.04 & 0.03 & \\
\hline V9360.... & 11.1033 & 41.3911 & 18.84 & 16.72 & 20.50 & 0.04 & 0.04 & 0.05 & \\
\hline V10916... & 11.1269 & 41.4099 & 19.13 & 16.78 & 22.44 & 0.12 & 0.19 & 0.15 & \\
\hline V11413... & 11.1367 & 41.3870 & 19.39 & 18.54 & 19.98 & 0.07 & 0.07 & 0.08 & \\
\hline V8969.... & 11.0983 & 41.3713 & 19.63 & 17.13 & 22.20 & 0.07 & 0.02 & 0.10 & \\
\hline V14370... & 11.1986 & 41.5138 & 19.72 & 16.69 & $\ldots$ & 0.12 & 0.06 & $\ldots$ & V4062 D31B \\
\hline V13441... & 11.1767 & 41.4471 & 19.94 & 16.53 & 21.00 & 0.23 & 0.20 & 0.06 & \\
\hline V5768.... & 11.0547 & 41.3761 & 20.03 & 19.40 & 20.39 & 0.09 & 0.08 & 0.04 & \\
\hline V6175 ... & 11.0605 & 41.3568 & 20.14 & 17.32 & 22.67 & 0.12 & 0.04 & 0.14 & \\
\hline V9115.... & 11.1004 & 41.3696 & 20.23 & 17.77 & 22.05 & 0.10 & 0.03 & 0.11 & \\
\hline V5588.... & 11.0488 & 41.4869 & 20.95 & 19.66 & 21.64 & 0.19 & 0.11 & 0.32 & \\
\hline V5283 ... & 11.0471 & 41.4166 & 21.02 & 19.01 & 22.30 & 0.41 & 0.11 & 0.17 & \\
\hline V433.... & 10.9907 & 41.3868 & 21.07 & 19.06 & 22.56 & 0.24 & 0.26 & 0.13 & \\
\hline V9205.... & 11.0987 & 41.4678 & 21.15 & 19.30 & 23.42 & 0.74 & 0.48 & 0.36 & \\
\hline V499 ..... & 10.9906 & 41.4050 & 21.16 & 19.53 & 22.33 & 0.24 & 0.17 & 0.11 & \\
\hline V14148... & 11.1968 & 41.3900 & 21.16 & 17.93 & $\ldots$ & 0.37 & 0.40 & $\ldots$ & \\
\hline V7581 ... & 11.0783 & 41.3585 & 21.26 & $\ldots$ & 22.24 & 0.45 & $\cdots$ & 0.41 & \\
\hline V3225 ... & 11.0230 & 41.3558 & 21.30 & 19.27 & 23.49 & 0.32 & 0.17 & 0.17 & \\
\hline V4241... & 11.0321 & 41.4616 & 21.66 & 18.41 & $\ldots$ & 0.34 & 0.09 & $\ldots$ & \\
\hline V8170... & 11.0830 & 41.4826 & 21.71 & $\ldots$ & 23.18 & 0.34 & $\ldots$ & 0.39 & \\
\hline V11642 ... & 11.1412 & 41.3844 & 21.74 & 19.89 & 23.04 & 0.31 & 0.12 & 0.35 & \\
\hline V4674 ... & 11.0385 & 41.4262 & 21.83 & 19.29 & 23.39 & 0.45 & 0.21 & 0.39 & \\
\hline V2346... & 11.0116 & 41.4046 & 21.87 & 19.86 & 23.38 & 0.46 & 0.39 & 0.25 & \\
\hline V8443.... & 11.0880 & 41.4442 & 21.95 & 20.38 & $\ldots$ & 0.35 & 0.27 & $\ldots$ & \\
\hline
\end{tabular}

Note. - Variables V14370 D31C was also found in Paper I. 
nine stars we have classified eight as Cepheids and one as an eclipsing binary (see Tables 1. 2 for cross-ids).

There was also by design a slight overlap between the M31C and M31B fields (Figure 9). There were four Cepheids from the M31C field in the overlap region, and they were all cross-identified in the M31B catalog, with very similar properties of their light curves (see Table 2). There was only one eclipsing binary in the overlap from the M31C field, V12594 D31C, and it was cross-identified as V2763 D31B, again with very similar properties of its light curve (see Table 1). We also cross-identified one miscellaneous variable (see Table 4 ), out of three detected in the M31B field and one detected in the $\mathrm{M} 31 \mathrm{C}$ field, which fell into the overlap region.

\section{Discussion}

In Figure 4 we show $V, V-I$ and $V, B-V$ color-magnitude diagrams for the variable stars found in the field M31C. The eclipsing binaries and Cepheids are plotted in the left panels and the other periodic variables and miscellaneous variables are plotted in the right panels. As expected, most of the eclipsing binaries occupy the blue upper main sequence of M31 stars, with the exception of the bright, probably foreground, W UMa system V13944 D31C. The Cepheid variables group near $B-V \sim 1.0$, with considerable scatter probably due to differential reddening across the field. The other periodic variable stars have positions on the CMD similar to the Cepheids. The miscellaneous variables are scattered throughout the CMDs and represent several classes of variability. Many of them are very red with $V-I>2.0$, and are probably Mira variables. Several brightest miscellaneous variables are probably foreground stars belonging to our Galaxy.

In Figure 9 we plot the location of eclipsing binaries and Cepheids in the fields $\mathrm{M} 31 \mathrm{C}$ and M31B, along with the blue stars $(B-V<0.4)$ selected from the photometric survey of M31 by Magnier et al. (1992) and Haiman et al. (1994). The sizes of the circles representing the Cepheids variables are proportional to the logarithm of their period. As could have been expected, both types of variables group along the spiral arms, as they represent relatively young populations of stars. We will explore various properties of our sample of Cepheids in the future paper (Sasselov et al. 1999, in preparation).

We would like to thank the TAC of the Michigan-Dartmouth-MIT (MDM) Observatory and the TAC of the F. L. Whipple Observatory (FLWO) for the generous amounts of telescope time devoted to this project. We are very grateful to Bohdan Paczyński for motivating us to undertake this project and his always helpful comments and suggestions. We thank Lucas Macri for taking some of the data described in this paper and Przemek Woźniak for his FITS-manipulation programs. The staff of the MDM and the FLWO observatories is thanked for their support during the long observing runs. KZS was supported by the Harvard-Smithsonian Center for Astrophysics Fellowship. JK was supported by NSF grant AST-9528096 to Bohdan Paczyński and by the Polish 
KBN grant 2P03D011.12. JLT was supported by the NSF grant AST-9401519.

\section{REFERENCES}

Andersen, J. 1991, A\&AR, 3, 91

Baade, W., Swope, H. H. 1963, AJ, 68, 435

Baade, W., Swope, H. H. 1965, AJ, 70, 212

Crotts, A. P. S., \& Tomaney, A. B. 1996, ApJ, 473, L87

Freedman, W. L., \& Madore, B. F. 1990, ApJ, 365, 186

Freedman, W. L., Wilson, C. D., \& Madore, B. F. 1991, ApJ, 372, 455

Gaposchkin, S. 1962, AJ, 67, 334

Haiman, Z., et al. 1994, A\&A, 286, 725

Hilditch, R. W. 1996, in: ASP Conf. Ser. 90, The Origins, Evolution and Destinies of Binary Stars in Clusters, ed. E. F. Milone \& J.-C. Mermilliod (San Francisco: ASP), 207

Holland, S. 1998, AJ, 115, 1916

Hubble, E. 1926, ApJ, 63, 236

Hubble, E. 1929, ApJ, 69, 103

Huterer, D., Sasselov, D. D., Schechter, P. L. 1995, AJ, 100, 2705

Jacoby, G. H., et al. 1992, PASP, 104, 599

Kaluzny, J., Stanek, K. Z., Krockenberger, M., Sasselov, D. D., Tonry, J. L., \& Mateo, M. 1998, AJ, 115, 1016 (Paper I)

Krockenberger, M., Sasselov, D. D., \& Noyes, R. 1997, ApJ, 479, 875

Lafler, J., \& Kinman, T. D. 1965, ApJS, 11, 216

Landolt, A. 1992, AJ, 104, 340

Magnier, E. A., Augusteijn, T., Prins, S., van Paradijs, J., \& Lewin, W. H. G. 1997, A\&AS, 126, 401 (Ma97)

Magnier, E. A., Lewin, W. H. G., Van Paradijs, J., Hasinger, G., Jain, A., Pietsch, W., \& Truemper, J. 1992, A\&AS, 96, 37

Metzger, M. R., Tonry, J. L., \& Luppino, G. A. 1993, in ASP Conf. Ser. 52, Astronomical Data Analysis Software and Systems II, ed. R. J. Hanisch, R. J. V. Brissenden, \& J. Barnes, (San Francisco: ASP), 300

Paczyński, B. 1997, in The Extragalactic Distance Scale, ed. M. Livio, M. Donahue \& N. Panagia (Cambridge: Cambridge Univ. Press), 273

Sasselov, D. D., et al. 1999, in preparation 
Stanek, K. Z., Kaluzny, J., Krockenberger, M., Sasselov, D. D., Tonry, J. L., \& Mateo, M. 1998, AJ, 115, 1894 (Paper II)

Stanek. K. Z., \& Garnavich, P. M. 1998, ApJ, 503, L131

Stetson, P.B. 1987, PASP, 99191

Stetson, P.B. 1992, in ASP Conf. Ser. 25, Astrophysical Data Analysis Software and Systems I, ed. D. M. Worrall, C. Bimesderfer, \& J. Barnes (San Francisco: ASP), 297

Stetson, P. B. 1996, PASP, 108, 851

Szentgyorgyi, A., et al. 1999, in preparation

Tonry, J. L., Blakeslee, J. P., Ajhar, E. A., \& Dressler, A., 1997, ApJ, 475, 399 


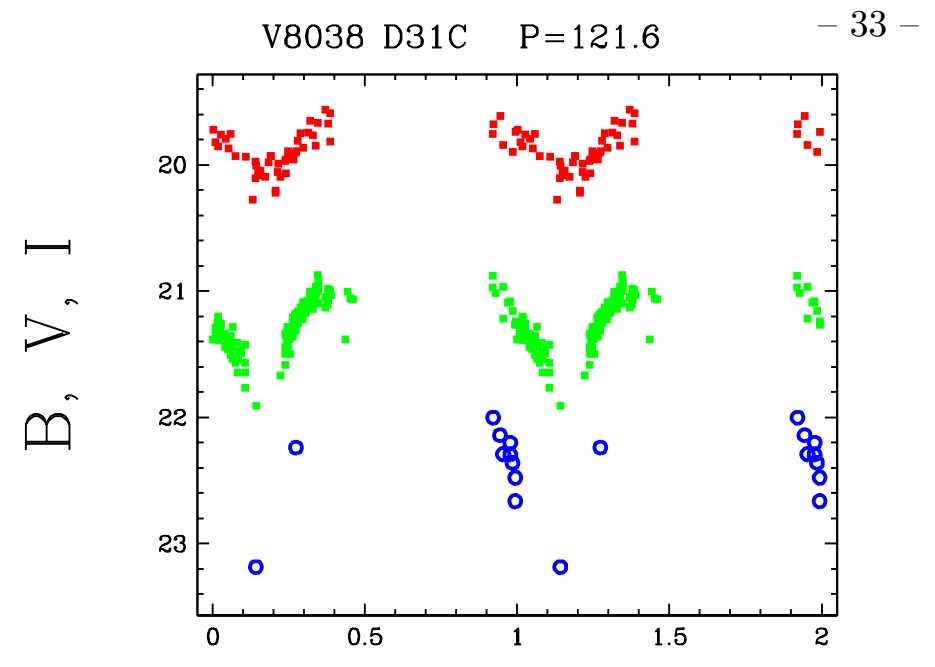

Phase

Fig. 6.- Continued.

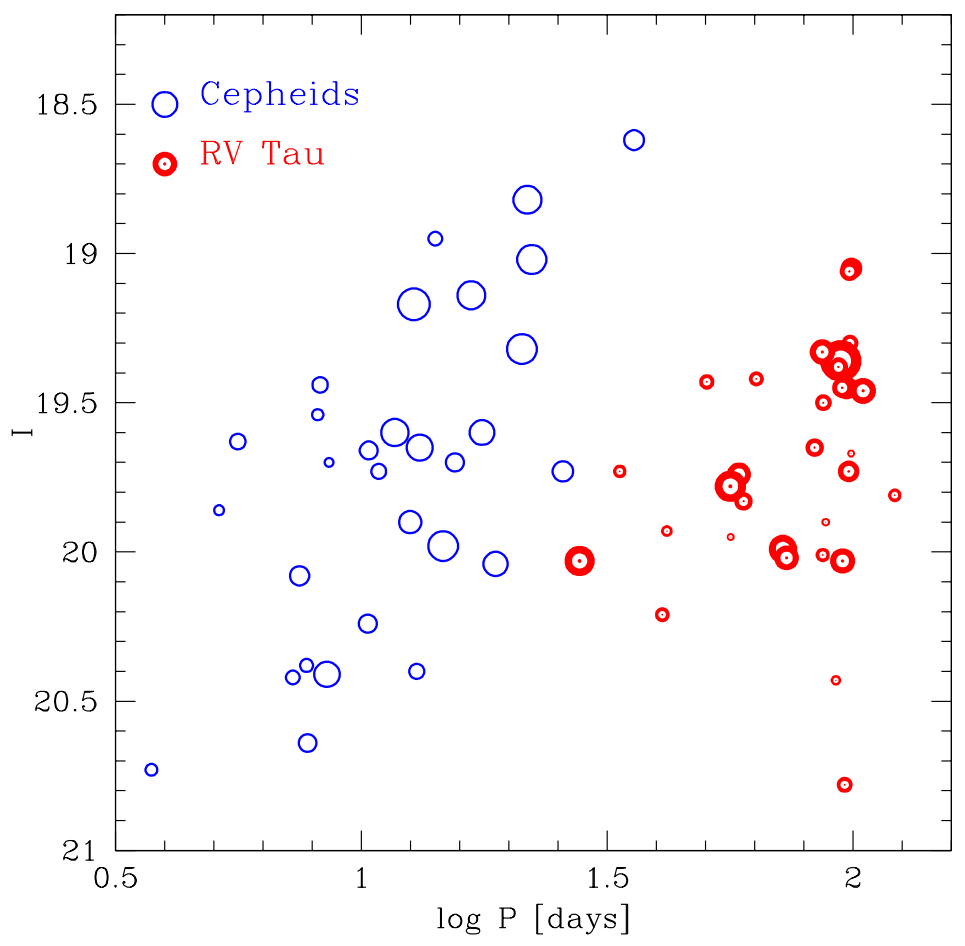

Fig. 7.- Diagram of $\log P$ vs. $I$ for the Cepheids (open circles) and RV Tau (dotted circles) variables. The sizes of the circles are proportional to the $V$ amplitude of the variability. 


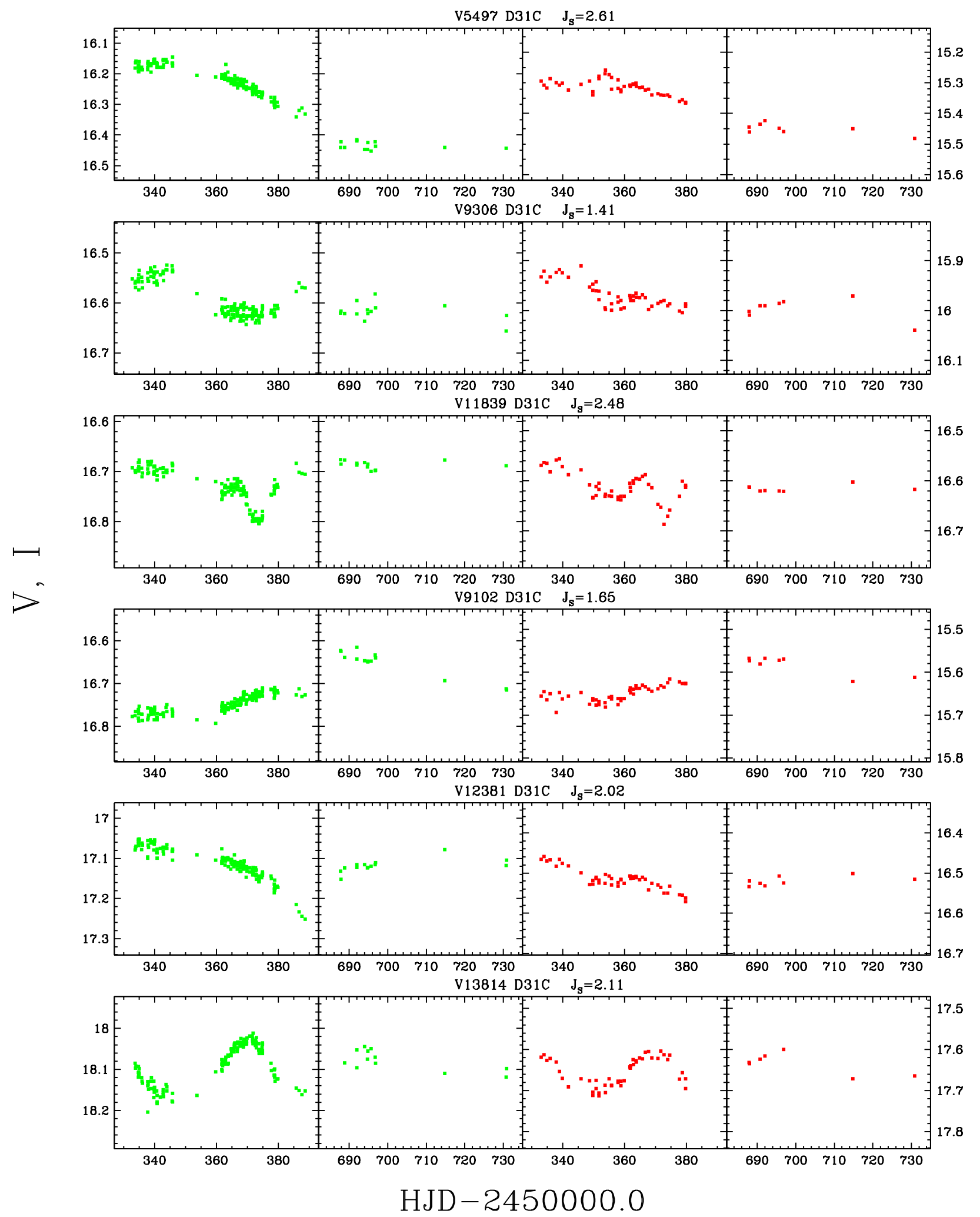

Fig. 8.- VI lightcurves of the miscellaneous variables found in the field M31C. I (if present) is plotted in the two right panels. $B$-band data is not shown. 


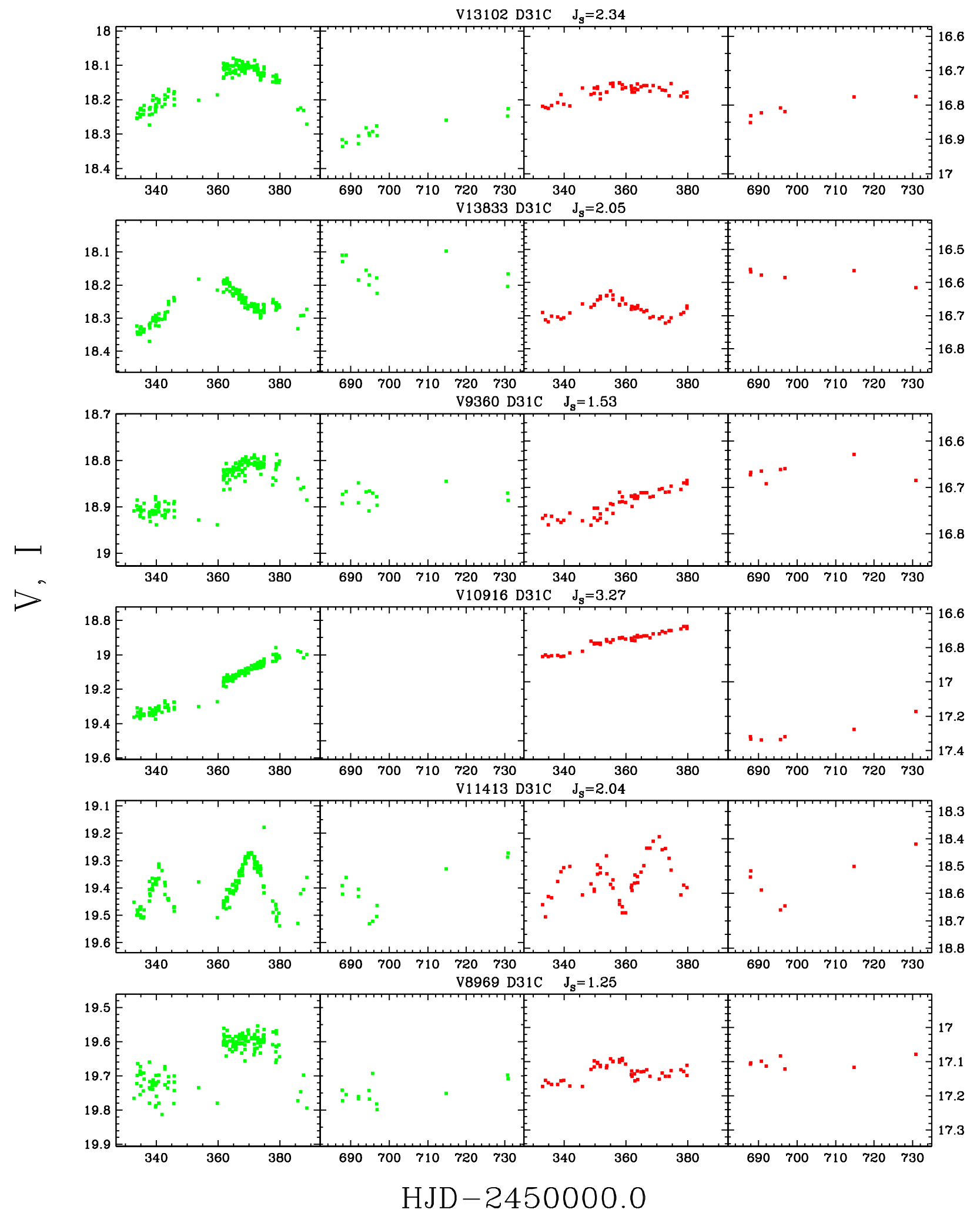

Fig. 8.- Continued. 


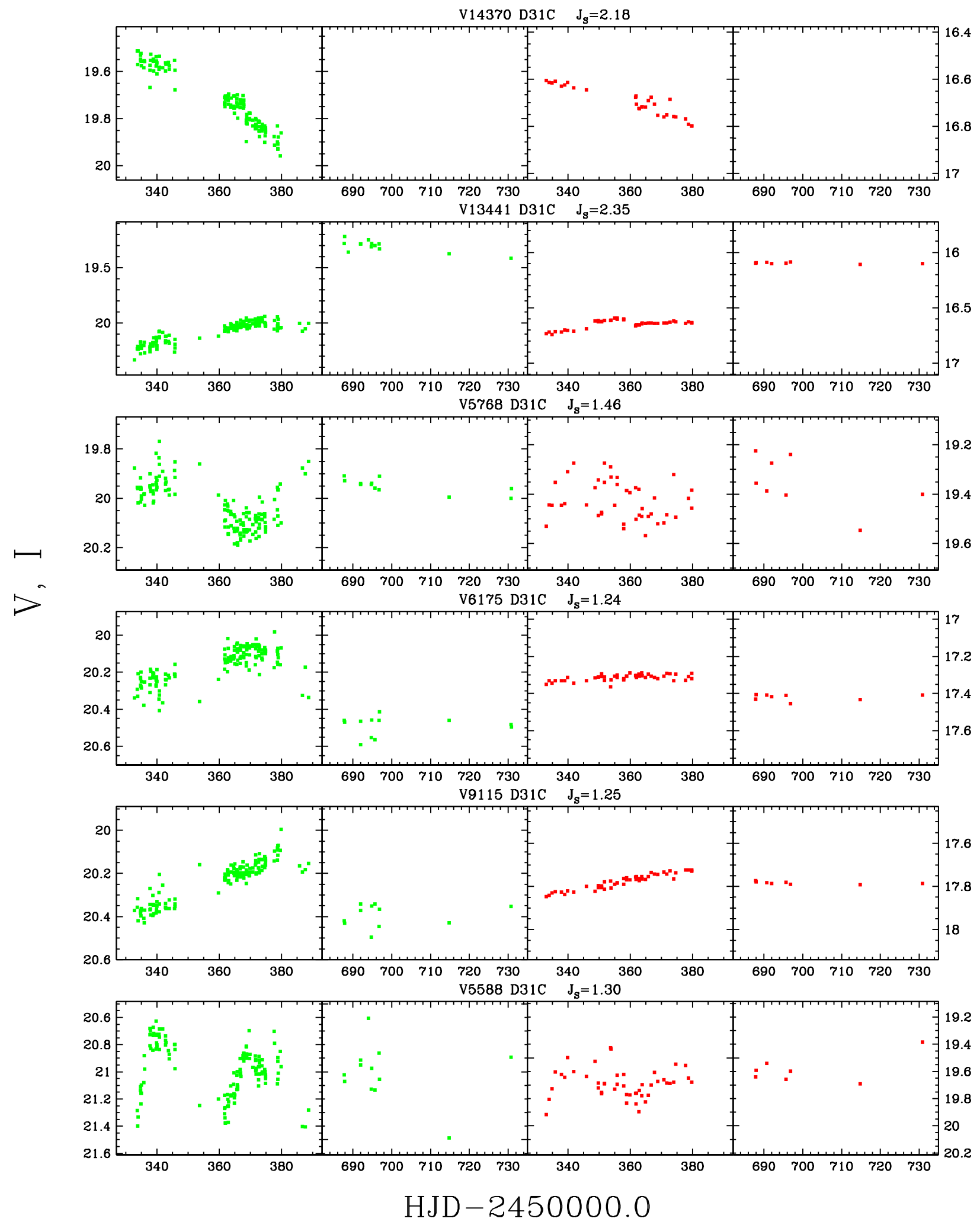

Fig. 8.- Continued. 

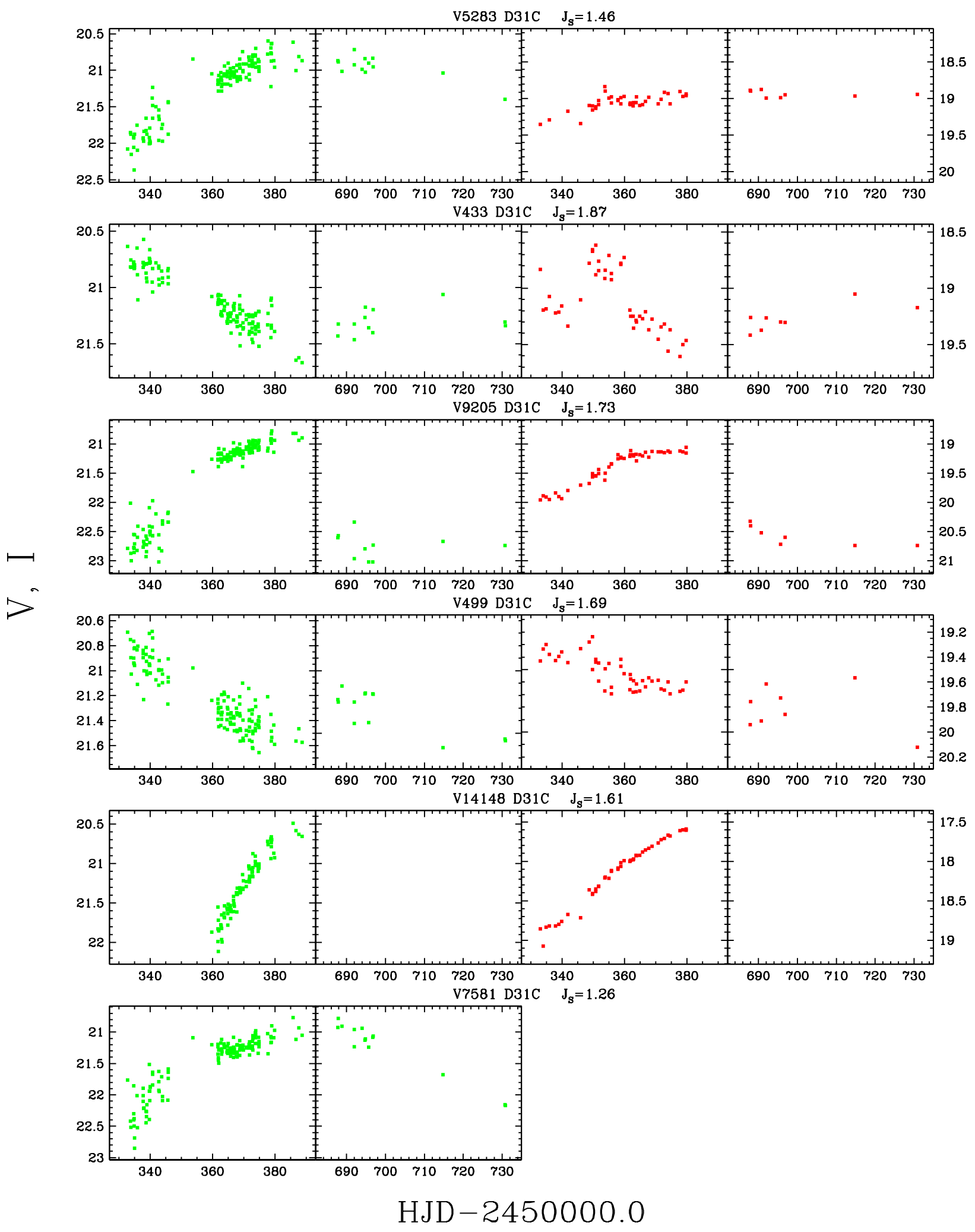

Fig. 8.- Continued. 


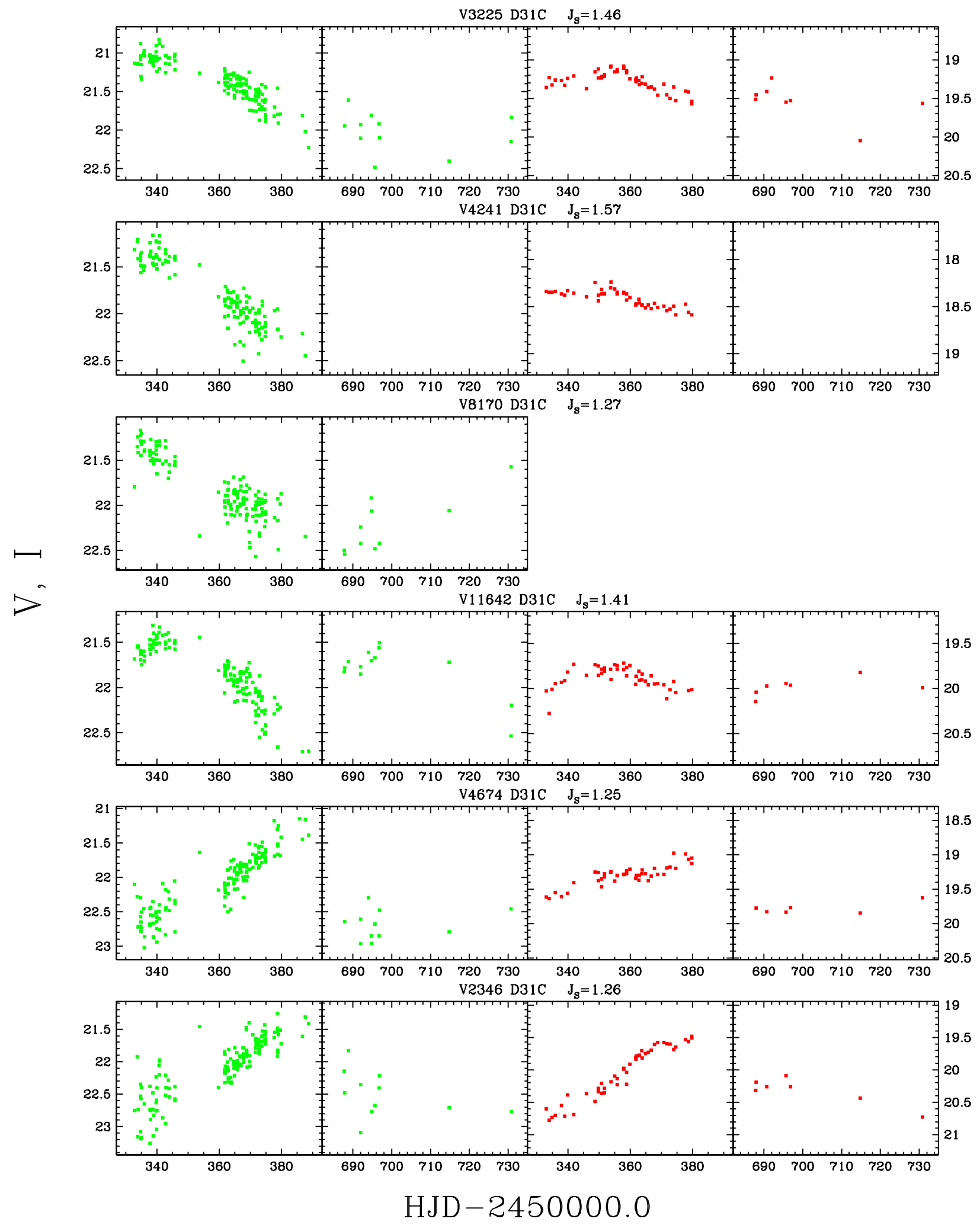

Fig. 8.- Continued. 


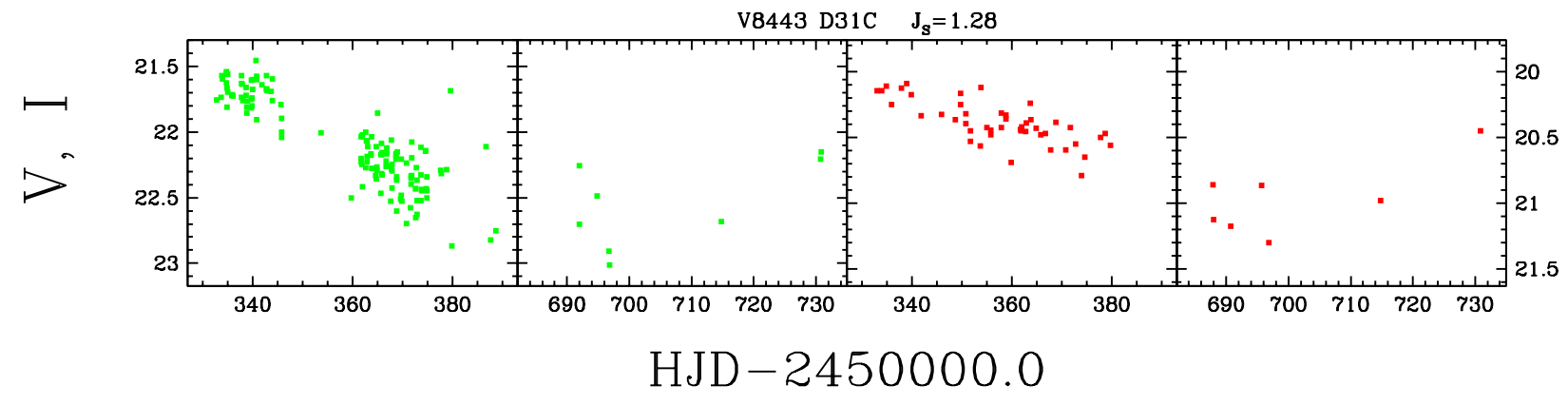

Fig. 8.- Continued.

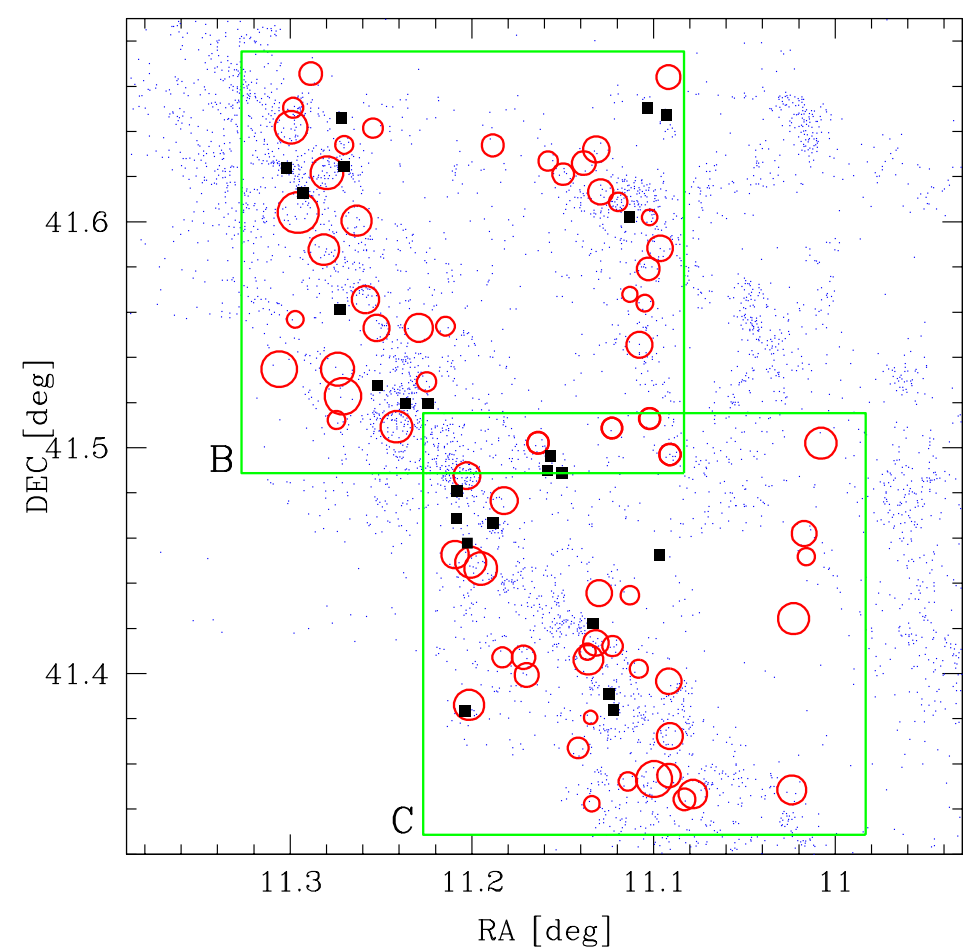

Fig. 9.- Location of eclipsing binaries (filled squares) and Cepheids (open circles) in the fields $\mathrm{M} 31 \mathrm{C}$ and M31B, along with the blue stars $(B-V<0.4)$ selected from the photometric survey of M31 by Magnier et al. (1992) and Haiman et al. (1994). The sizes of the circles representing the Cepheids variables are proportional to the logarithm of their period. 